\title{
Two Beyond-Primitive Extrasolar Planetesimals
}

\author{
S. Xu(许偲艺 $)^{a}$, M. Jura ${ }^{a}$, B. Klein ${ }^{a}$, D. Koester ${ }^{b}$, B. Zuckerman ${ }^{a}$
}

\begin{abstract}
Using the Cosmic Origins Spectrograph onboard the Hubble Space Telescope, we have obtained high-resolution ultraviolet observations of GD 362 and PG 1225079, two helium-dominated, externally-polluted white dwarfs. We determined or placed useful upper limits on the abundances of two key volatile elements, carbon and sulfur, in both stars; we also constrained the zinc abundance in PG 1225-079. In combination with previous optical data, we find strong evidence that each of these two white dwarfs has accreted a parent body that has evolved beyond primitive nebular condensation. The planetesimal accreted onto GD 362 had a bulk composition roughly similar to that of a mesosiderite meteorite based on a reduced chi-squared comparison with solar system objects; however, additional material is required to fully reproduce the observed mid-infrared spectrum for GD 362. No single meteorite can reproduce the unique abundance pattern observed in PG 1225-079; the best fit model requires a blend of ureilite and mesosiderite material. From a compiled sample of 9 well-studied polluted white dwarfs, we find evidence for both primitive planetesimals, which are a direct product from nebular condensation, as well as beyond-primitive planetesimals, whose final compositions were mainly determined by post-nebular processing.
\end{abstract}

Subject headings: planetary systems stars: abundances white dwarfs

\section{INTRODUCTION}

Planetesimals are building blocks of planets and their formation is a key step towards planet formation. How do planetesimals form? What determines their bulk composition? To answer these questions, we start by examining our own solar system.

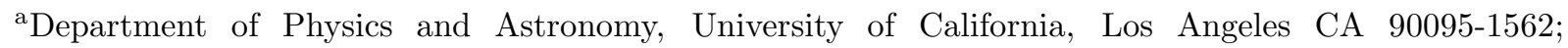
sxu@astro.ucla.edu, jura@astro.ucla.edu, kleinb@astro.ucla.edu, ben@astro.ucla.edu

${ }^{b}$ Institut fur Theoretische Physik und Astrophysik, University of Kiel, 24098 Kiel, Germany; koester@astrophysik.uni-kiel.de
} 
The overall configuration of the solar system is that volatile-depleted, dry rocky objects are ubiquitous relatively close to the Sun while volatile-rich, icy objects are found beyond the snow line. This correlation between the volatile fraction and heliocentric distance can be explained by primitive nebular condensation: refractory elements condensed closer to the Sun while volatile elements can only be incorporated into the planetesimals where the temperature is low enough. Many solar system objects have experienced some additional processing that changed their initial compositions. For example, it has been argued that a collision between a large asteroid and proto-Mercury stripped off most of Mercury's silicate mantle, leaving it $\sim 70 \%$ iron by mass (Benz et al. 1988). Also, the "late veneer" has delivered a large amount of water and volatiles onto Earth (Chyba 1990). Post-nebular processing, such as collisions, melting and differentiation, is important in redistributing the elements among solar system objects.

Currently, the best way to measure the elemental compositions of planetesimals in the solar system is from meteorites, which are fragments from collisions among asteroids. Following O’Neill \& Palme (2008), we classify all meteorites into two categories in this paper. (i) "Chondritic" is used to refer to chondrites, which are a direct product of nebular processing. Objects in this category are described as "primitive" planetesimals. (ii) "Non-chondritic" objects consist of achondrites, stoney-iron meteorites and iron meteorites. Examples of their parent bodies include the Moon, Mars or asteroids that have experienced various amounts of post-nebular processing. Planetesimals in this category are considered to be "beyondprimitive".

What about planetesimal formation in extrasolar planetary systems? High-resolution, high-sensitivity spectroscopic observations of externally-polluted white dwarfs are a powerful tool for determining the bulk elemental compositions of extrasolar planetesimals (Jura 2013). Calculations show that minor planets can survive the red giant stage of a star and persist into the white dwarf phase with most of their internal water and volatiles intact (Jura 2008; Jura \& Xu 2010). Orbital perturbations from one or multiple planets can cause these planetesimals to stray into the tidal radius of the white dwarf and get tidally disrupted (Debes \& Sigurdsson 2002; Bonsor et al. 2011; Debes et al. 2012), sometimes producing a dust disk that emits mostly in the infrared (Jura 2003; Kilic et al. 2006; von Hippel et al. 2007; Farihi et al. 2009; Xu \& Jura 2012). Eventually, all this planetary debris is accreted onto the central white dwarf and pollutes its otherwise pure hydrogen or helium atmosphere.

The first comprehensive abundance measurement of an externally-polluted white dwarf was performed by Zuckerman et al. (2007), who identified 15 elements heavier than helium in the atmosphere of GD 362, including Mg, Si and Fe, which are often called the "common elements" (Larimer 1988). The disrupted object had a minimum mass $\sim 10^{22} \mathrm{~g}$, which 
is comparable to that of a massive solar system asteroid. Three years later, the abundances of eight heavy elements were determined in the atmosphere of GD 40, including all the major rock-forming elements - O, Mg, Si and Fe (Klein et al. 2010). Now there are many more high-resolution optical spectroscopic studies of externally-polluted white dwarfs [e.g., Klein et al. (2011); Melis et al. (2011); Zuckerman et al. (2011); Farihi et al. (2011); Dufour et al. (2012); Vennes et al. (2010, 2011)].

However, optical spectroscopy of externally-polluted white dwarfs typically does not enable sensitive detection of highly-volatile elements, such as carbon, nitrogen and sulfur, which are key to understanding the thermal history of the system. Ultraviolet spectroscopy is complimentary to optical observations in determination of volatile abundances.

To-date, there are four white dwarfs with both published high-resolution optical and ultraviolet measurement:1; we are beginning to accumulate an atlas of the compositions of extrasolar planetesimals. To zeroth order, we find that they are strikingly similar to meteorites in the solar system: (i) $\mathrm{O}, \mathrm{Mg}$, Si and $\mathrm{Fe}$ are always dominant and their sum is more than $85 \%$ of the accreted mass; (2) volatile elements, especially C, are typically depleted by more than a factor of 10 compared to solar abundances 2 .

In this paper, we report ultraviolet spectroscopic observations of GD 362 and PG 1225079 with the Cosmic Origins Spectrograph (COS) onboard the Hubble Space Telescope (HST), complimentary to previous optical studies from the Keck High Resolution Echelle Spectrometer (HIRES) (Zuckerman et al. 2007; Klein et al. 2011). PG 1225-079 has been observed with the low-resolution International Ultraviolet Explorer (IUE) (Wolff et al. 2002); there is no previous ultraviolet spectroscopy for GD 362. The rest of the paper is organized as follows. Data reduction is summarized in section 2 and atmospheric abundance determinations are reported in section 3. In section 4, we used a reduced chi-squared analysis to look for solar system analogs to the accreted parent bodies. The formation mechanisms of extrasolar planetesimals are assessed in section 5 and conclusions are given in section 6 . In Appendix A, we report the Herschel Photodetecting Array Camera and Spectrometer (PACS) observation of GD 362. In Appendix B, we extend the reduced chi-squared analysis to two additional externally-polluted helium white dwarfs with both high-resolution optical and ultraviolet observations.

\footnotetext{
${ }^{1}$ The four white dwarfs are: GD 61 (Desharnais et al. 2008; Farihi et al. 2011); GD 40, G241-6 (Klein et al. 2010, 2011; Zuckerman et al. 2010; Jura et al. 2012) and WD 1929+012 (Vennes et al. 2010, 2011; Melis et al. 2011; Gänsicke et al. 2012).

${ }^{2}$ Very recently, Koester et al. (2012) reported several white dwarfs with solar carbon-to-silicon ratio. However, the source of this pollution is unclear and more analysis is forthcoming.
} 


\section{OBSERVATIONS AND DATA REDUCTION}

GD 362 and PG 1225-079 were observed during HST/COS Cycle 18 under program 12290. These two white dwarfs are too cool to be observed effectively with the G130M grating centering around $1300 \AA$, as was employed by Jura et al. (2012) and Gänsicke et al. (2012) for other hotter white dwarfs. Instead, the G185M grating was used with a central wavelength of $1921 \AA$ and wavelength coverage of $1800-1840 \AA, 1903-1940 \AA$ and 2008 $2044 \AA$. The spectral resolution was $\sim 18,000$. Total exposure times were 7411 and 1805 sec for GD 362 and PG 1225-079, respectively.

The raw data were processed using the standard pipeline CALCOS 2.13.6. The fluxes at $2030 \AA$ are $2.9 \times 10^{-15} \mathrm{erg} \mathrm{s}^{-1} \mathrm{~cm}^{-2} \AA^{-1}$ and $1.5 \times 10^{-14} \mathrm{erg} \mathrm{s}^{-1} \mathrm{~cm}^{-2} \AA^{-1}$ for GD 362 and PG 1225-079, respectively, in approximate agreement with broadband NUV fluxes from the GALEX satellite. The signal-to-noise ratio (SNR) in the original un-smoothed spectrum was 6 for PG 1225-079 and 4 for GD 362.

Following previous data reduction procedures (Klein et al. 2010, 2011; Jura et al. 2012), for PG 1225-079, equivalent widths (EWs) of each spectral line were measured in the unsmoothed spectra by fitting a Voigt profile with three different nearby continuum intervals in IRAF. The EW uncertainty is calculated by adding the standard deviation of the three EWs and the average uncertainty from the profile fitting in quadrature. The EW upper limit is obtained by artificially inserting a spectral line with different abundance into the model and comparing with the data. We adopt a different method to measure the EW for C I 1930.9 $\AA$ in GD 362, as described in section 3.1. The measured values are listed in Tables 1 and 2 for GD 362 and PG 1225-079, respectively. The average Doppler shift relative to the Sun for PG 1225-079 is $42 \pm 13 \mathrm{~km} \mathrm{~s}^{-1}$, in essential agreement with the value $49 \pm 3$ $\mathrm{km} \mathrm{s}^{-1}$ derived from optical studies (Klein et al. 2011). The large velocity dispersion in the ultraviolet is due to the low SNR of the spectrum and the $\sim 15 \mathrm{~km} \mathrm{~s}^{-1}$ uncertainty of COS (COS Instrument Handbook). For GD 362, we marginally detected C I $1930.9 \AA$ and it has a Doppler shift of $48 \mathrm{~km} \mathrm{~s}^{-1}$, in agreement with $49.3 \pm 1.0 \mathrm{~km} \mathrm{~s}^{-1}$ from the optical study (Zuckerman et al. 2007).

\section{ATMOSPHERIC ABUNDANCE DETERMINATIONS}

Because we are most interested in the abundance of an element relative to other heavy elements and these ratios are not strongly dependent upon the stellar temperature and surface gravity (Klein et al. 2011), we only adopt one set of stellar parameters as listed in Table 3 and compute the model spectra following Koester (2010). Atomic data are mostly 
Table 1: Measured Equivalent Widths and Abundance Determinations for GD 362

\begin{tabular}{lcccc}
\hline \hline Ion & $\begin{array}{c}\lambda \\
(\AA)\end{array}$ & $\begin{array}{c}\mathrm{E}_{\text {low }} \\
(\mathrm{eV})\end{array}$ & $\begin{array}{c}\mathrm{EW} \\
(\mathrm{m} \AA)\end{array}$ & $\log \mathrm{n}(\mathrm{Z}) / \mathrm{n}(\mathrm{He})$ \\
\hline C I & 1930.905 & 1.26 & $560_{-158}^{+230} a$ & $-6.70 \pm 0.30$ \\
& & & & \\
S I & 1807.311 & 0 & $\lesssim 900$ & $\lesssim-6.70$ \\
S I & 1820.341 & 0.049 & $\lesssim 710$ & $\lesssim-6.40$ \\
S & & & & $\lesssim-6.70$ \\
\hline
\end{tabular}

${ }^{a}$ This is measured from the model spectra, as described in section 3.1.

taken from the Vienna Atomic Line Database (Kupka et al. 1999). The computed model atmosphere spectra were convolved with the COS NUV line spread function 3 . The abundance of each element was derived by comparing the EW of each spectral line with the value derived from the model atmosphere, as shown in Figures 1 - 5 and Tables 11 and 2. The final abundances, combining ultraviolet with optical observations, are given in Tables 4 and 5 for GD 362 and PG 1225-079, respectively. Our results mostly agree with previous reports but have a higher accuracy. For PG 1225-079, we newly derive the abundances of carbon and silicon and have tentative detections of sulfur and zinc. The magnesium abundance is updated while the iron abundance agrees with previous optical results. Because the data are noisier for GD 362, we are only able to crudely constrain the abundance of carbon and sulfur.

\subsection{Carbon}

There is only one useful carbon line in the observed wavelength interval, C I 1930.9 $\AA$, as shown in Figures 11 and 2, Because it arises from an excited level, it cannot be contaminated by interstellar absorption. However, this line can be blended with Mn II 1931.4 A. Fortunately, accurate Mn abundances have been determined for both stars from optical data (Zuckerman et al. 2007; Klein et al. 2011) and the predicted EW for Mn II $1931.4 \AA$ is less than $50 \mathrm{~m} \AA$ in the model spectrum. Considering the measured EW of this feature is more than $500 \mathrm{~m} \AA$ for both stars (see Tables 1 and 2), we conclude that the line

\footnotetext{
${ }^{3}$ http://www.stsci.edu/hst/cos/performance/spectral_resolution/nuv_model_lsf
} 
Table 2: Measured Equivalent Widths and Abundance Determinations for PG 1225-079

\begin{tabular}{lcccc}
\hline \hline & & & & \\
Ion & $\lambda$ & $\mathrm{E}_{\text {low }}$ & $\mathrm{EW}$ & $\log \mathrm{n}(\mathrm{Z}) / \mathrm{n}(\mathrm{He})$ \\
& $(\AA)$ & $(\mathrm{eV})$ & $(\mathrm{mA})$ & \\
\hline C I & 1930.905 & 1.26 & $1600 \pm 200$ & $-7.80 \pm 0.10$ \\
& & & & \\
S I & 1807.311 & 0 & $\lesssim 170$ & $\lesssim-9.50$ \\
S I & 1820.341 & 0.049 & $\lesssim 150$ & $\lesssim-9.30$ \\
S & & & & $\lesssim-9.50$ \\
& & & & $\vdots-7.60$ \\
Mg I & $2026.477^{a}$ & 0 & $288 \pm 100^{b}$ & \\
& & & & $-7.44 \pm 0.10$ \\
Si II & 1808.013 & 0 & $936 \pm 109$ & $-7.46 \pm 0.10$ \\
Si II & 1816.928 & 0.04 & $1232 \pm 145$ & $-7.45 \pm 0.10$ \\
Si & & & & $-7.62 \pm 0.28$ \\
& & & & $-7.35 \pm 0.24$ \\
Fe II & 1925.987 & 2.52 & $192 \pm 72$ & \\
Fe II & 2011.347 & 2.58 & $309 \pm 97$ & -11.30 \\
Fe II & 2019.429 & 1.96 & $211 \pm 67$ & $-7.56 \pm 0.24$ \\
Fe II & 2021.402 & 1.67 & $181 \pm 68$ & $-7.71 \pm 0.27$ \\
Fe II & 2033.061 & 2.03 & $311 \pm 67$ & $-7.24 \pm 0.17$ \\
Fe II & 2041.345 & 1.964 & $215 \pm 50$ & $-7.24 \pm 0.18$ \\
Fe & & & & -7.45 \\
& & & & \\
Zn II & 2026.136 & 0 & $288 \pm 47^{b}$ & \\
\hline
\end{tabular}

${ }^{a}$ The atomic parameters for this line are taken from Kelleher \& Podobedova (2008).

${ }^{b} \mathrm{Mg}$ I $2026.5 \AA$ and Zn II $2026.1 \AA$ are blended and the reported EW is for the entire feature. 
Table 3: Adopted Stellar Properties

\begin{tabular}{lcccccc}
\hline \hline star & $\begin{array}{c}\mathrm{M}_{*} \\
\left(\mathrm{M}_{\odot}\right)\end{array}$ & $\begin{array}{c}\mathrm{T} \\
(\mathrm{K})\end{array}$ & $\begin{array}{c}\log \mathrm{g} \\
\left(\mathrm{cm}^{2} \mathrm{~s}^{-1}\right)\end{array}$ & $\begin{array}{c}\mathrm{D} \\
(\mathrm{pc})\end{array}$ & $\log \mathrm{M}_{c v z} / \mathrm{M}_{*}{ }^{a}$ & Ref \\
\hline GD 362 & 0.72 & 10,540 & 8.24 & 51 & -6.71 & $(1)(2)$ \\
PG 1225-079 & 0.58 & 10,800 & 8.00 & 26 & -5.02 & $(3)(4)$ \\
\hline
\end{tabular}

${ }^{a}$ Newly-derived mass of the convective zone (see section 4).

References. (1) Kilic et al. (2008); (2) Zuckerman et al. (2007); (3) Klein et al. (2011); (4) Farihi et al. (2005).

is dominated by C I $1930.9 \AA$. For PG 1225-079, our derived carbon abundance屯 $[\mathrm{C}] /[\mathrm{He}]=$ $-7.80 \pm 0.10$ agrees with the IUE upper limit of -7.5 (Wolff et al. 2002).

For GD 362, the largest uncertainty is from the low SNR of the data; the measured continuum flux is $(3.1 \pm 1.0) \times 10^{-15} \mathrm{erg} \mathrm{s}^{-1} \mathrm{~cm}^{-2} \AA^{-1}$. It is hard to measure the EW of $\mathrm{C}$ I $1930.9 \AA$ directly from the noisy data. Instead, we computed model spectra with different carbon abundance to match the observed spectrum. In Figure 1, we present three best-fit models with $[\mathrm{C}] /[\mathrm{He}]=-6.4,[\mathrm{C}] /[\mathrm{He}]=-6.7,[\mathrm{C}] /[\mathrm{He}]=-7.0$ and a continuum flux at 4.1 $\times 10^{-15} \mathrm{erg} \mathrm{s}^{-1} \mathrm{~cm}^{-2} \AA^{-1}, 3.1 \times 10^{-15} \mathrm{erg} \mathrm{s}^{-1} \mathrm{~cm}^{-2} \AA^{-1}, 2.1 \times 10^{-15} \mathrm{erg} \mathrm{s}^{-1} \mathrm{~cm}^{-2} \AA^{-1}$, respectively. The final abundance is $[\mathrm{C}] /[\mathrm{He}]=-6.7 \pm 0.3$ and the EW reported in Table 1 is measured from the model spectra.

\subsection{Sulfur}

There are two useful sulfur lines, S I 1807.3 $\AA$ and S I $1820.3 \AA$. However, at best, we have only a tentative detection of sulfur in each star. S I $1807.3 \AA$, the stronger line, is adjacent to Si II 1808.0 A. Fortunately, for GD 362, the silicon abundance is determined from previous optical data (Zuckerman et al. 2007); for PG 1225-079, other ultraviolet lines can be used to derive the silicon abundance (see section 3.4). The data and model atmosphere spectra for GD 362 and PG 1225-079 are presented in Figures 3 and 4, respectively. Considering the apparent match between the model and data for both S I lines, tentative sulfur abundances of -6.7 for GD 362 and -9.5 for PG 1225-079 can be assigned. Conservatively, these results

\footnotetext{
${ }^{4}$ Here, $\log \mathrm{n}(\mathrm{X}) / \mathrm{n}(\mathrm{Y})$ is abbreviated as $[\mathrm{X}] /[\mathrm{Y}]$.
} 
Table 4: Atmospheric Abundances for GD 362

\begin{tabular}{lllc}
\hline \hline $\mathrm{Z}$ & $\log \mathrm{n}(\mathrm{Z}) / \mathrm{n}(\mathrm{He})^{a}$ & $\begin{array}{l}\mathrm{t}_{\text {set }}^{b} \\
\left(10^{5} \mathrm{yr}\right)\end{array}$ & $\begin{array}{c}\dot{M}\left(\mathrm{Z}_{i}\right)^{c} \\
\left(\mathrm{~g} \mathrm{~s}^{-1}\right)\end{array}$ \\
\hline $\mathrm{H}$ & $-1.14 \pm 0.10$ & $\ldots$ & $\cdots$ \\
$\mathrm{C}^{*}$ & $-6.70 \pm 0.30$ & 2.1 & $2.5 \times 10^{7}$ \\
$\mathrm{~N}$ & $<-4.14$ & 2.2 & $<9.0 \times 10^{9}$ \\
$\mathrm{O}$ & $<-5.14$ & 2.2 & $<1.1 \times 10^{9}$ \\
$\mathrm{Na}$ & $-7.79 \pm 0.20$ & 2.2 & $3.7 \times 10^{6}$ \\
$\mathrm{Mg}$ & $-5.98 \pm 0.25$ & 2.2 & $2.5 \times 10^{8}$ \\
$\mathrm{Al}$ & $-6.40 \pm 0.20$ & 1.6 & $1.5 \times 10^{8}$ \\
$\mathrm{Si}$ & $-5.84 \pm 0.30$ & 1.2 & $7.2 \times 10^{8}$ \\
$\mathrm{~S}$ & $\lesssim-6.70^{d}$ & 0.79 & $\lesssim 1.7 \times 10^{8}$ \\
$\mathrm{Ca}$ & $-6.24 \pm 0.10$ & 0.99 & $5.1 \times 10^{8}$ \\
$\mathrm{Sc}$ & $-10.19 \pm 0.30$ & 0.93 & $6.8 \times 10^{4}$ \\
$\mathrm{Ti}$ & $-7.95 \pm 0.10$ & 0.94 & $1.2 \times 10^{7}$ \\
$\mathrm{~V}$ & $-8.74 \pm 0.30$ & 0.95 & $2.1 \times 10^{6}$ \\
$\mathrm{Cr}$ & $-7.41 \pm 0.10$ & 1.0 & $4.3 \times 10^{7}$ \\
$\mathrm{Mn}$ & $-7.47 \pm 0.10$ & 1.0 & $4.0 \times 10^{7}$ \\
$\mathrm{Fe}$ & $-5.65 \pm 0.10$ & 1.1 & $2.5 \times 10^{9}$ \\
$\mathrm{Co}$ & $-8.50 \pm 0.40$ & 0.99 & $4.1 \times 10^{6}$ \\
$\mathrm{Ni}$ & $-7.07 \pm 0.15$ & 1.0 & $1.1 \times 10^{8}$ \\
$\mathrm{Cu}$ & $-9.20 \pm 0.40$ & 0.83 & $1.1 \times 10^{6}$ \\
$\mathrm{Sr}$ & $-10.42 \pm 0.30$ & 0.56 & $1.3 \times 10^{5}$ \\
$\mathrm{Total}$ & & & $4.4 \times 10^{9}$ \\
\hline & & &
\end{tabular}

* New measurements from this paper. The rest are from Zuckerman et al. (2007) but we reference abundances relative to He, the dominant element in GD 362's atmosphere, rather than $\mathrm{H}$, as presented in Zuckerman et al. (2007). Consequently, there is a possible systematic offset up to 0.1 dex in all entries derived from that paper.

${ }^{a}$ The final abundance of an element combining optical and ultraviolet data.

${ }^{b}$ Newly-derived settling times in the convective zone (see section 4); they are typically a factor of 2-3 longer than previously-derived values in Koester (2009).

${ }^{c}$ Accretion rates calculated from Equation (1).

${ }^{d}$ The equality sign corresponds to the red model fit shown in figures. 
Table 5: Atmospheric Abundances for PG 1225-079

\begin{tabular}{lccc}
\hline \hline $\mathrm{Z}$ & $\log \mathrm{n}(\mathrm{Z}) / \mathrm{n}(\mathrm{He})$ & $\begin{array}{c}\mathrm{t}_{\text {set }} \\
\left(10^{6} \mathrm{yr}\right)\end{array}$ & $\begin{array}{c}\dot{M}\left(\mathrm{Z}_{i}\right) \\
\left(\mathrm{g} \mathrm{s}^{-1}\right)\end{array}$ \\
\hline $\mathrm{H}$ & $-4.05 \pm 0.10$ & $\ldots$ & $\ldots$ \\
$\mathrm{C}^{*}$ & $-7.80 \pm 0.10$ & 5.5 & $3.1 \times 10^{6}$ \\
$\mathrm{O}$ & $<-5.54$ & 4.5 & $<9.1 \times 10^{8}$ \\
$\mathrm{Na}$ & $<-8.26$ & 4.4 & $<2.6 \times 10^{6}$ \\
$\mathrm{Mg} *$ & $-7.50 \pm 0.20$ & 4.8 & $1.4 \times 10^{7}$ \\
$\mathrm{Al}$ & $<-7.84$ & 3.6 & $<9.5 \times 10^{6}$ \\
$\mathrm{Si}^{*}$ & $-7.45 \pm 0.10$ & 3.0 & $3.0 \times 10^{7}$ \\
$\mathrm{~S}^{*}$ & $\lesssim-9.50$ & 1.7 & $\lesssim 5.2 \times 10^{5}$ \\
$\mathrm{Ca}$ & $-8.06 \pm 0.03$ & 1.9 & $1.6 \times 10^{7}$ \\
$\mathrm{Sc}$ & $-11.29 \pm 0.07$ & 1.8 & $1.1 \times 10^{4}$ \\
$\mathrm{Ti}$ & $-9.45 \pm 0.02$ & 1.8 & $8.3 \times 10^{5}$ \\
$\mathrm{~V}$ & $-10.41 \pm 0.10$ & 1.8 & $9.6 \times 10^{4}$ \\
$\mathrm{Cr}$ & $-9.27 \pm 0.06$ & 1.9 & $1.3 \times 10^{6}$ \\
$\mathrm{Mn}$ & $-9.79 \pm 0.14$ & 2.0 & $4.0 \times 10^{5}$ \\
$\mathrm{Fe}$ & $-7.42 \pm 0.07$ & 2.1 & $9.0 \times 10^{7}$ \\
$\mathrm{Ni}$ & $-8.76 \pm 0.14$ & 2.3 & $4.0 \times 10^{6}$ \\
$\mathrm{Zn}$ & $\lesssim-11.30$ & 2.2 & $\lesssim 1.3 \times 10^{4}$ \\
$\mathrm{Sr}$ & $<-11.65$ & 1.2 & $<1.4 \times 10^{4}$ \\
$\mathrm{Total}$ & & & $1.6 \times 10^{8}$ \\
\hline
\end{tabular}

${ }^{*}$ New results from this paper. The rest are from Klein et al. (2011).

Notes. The columns are defined the same as Table 4 . 


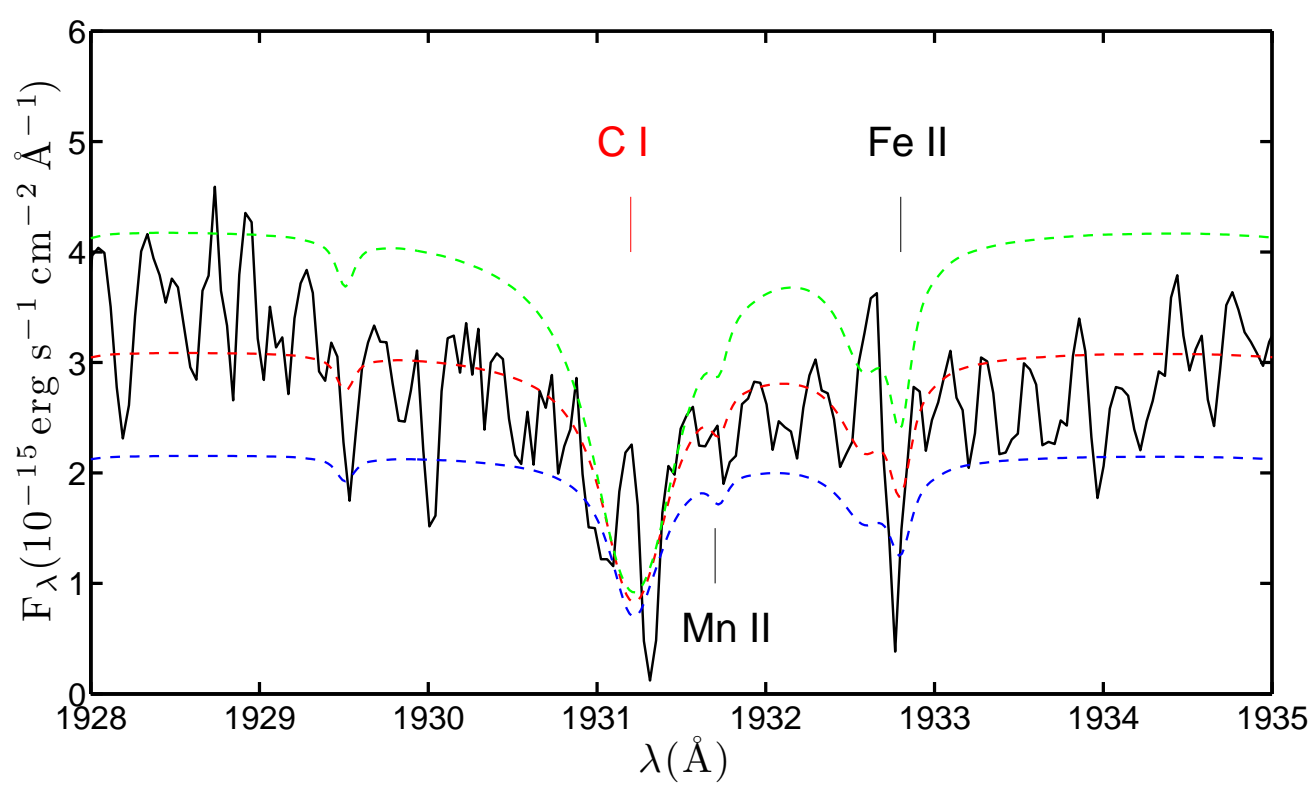

Fig. 1.-HST/COS spectrum of GD 362. The black line is the data smoothed with a 3 pixel boxcar. The green, red, blue line represents the computed model spectrum with $[\mathrm{C}] /[\mathrm{He}]=$ $-6.4,-6.7,-7.0$, respectively, placed at a different continuum level; the abundances of other elements are from Table 4. The adopted carbon abundance is $-6.7 \pm 0.3$. The red labels represent lines that are used for abundance determinations. Wavelength is presented in the star's reference frame in vacuum.

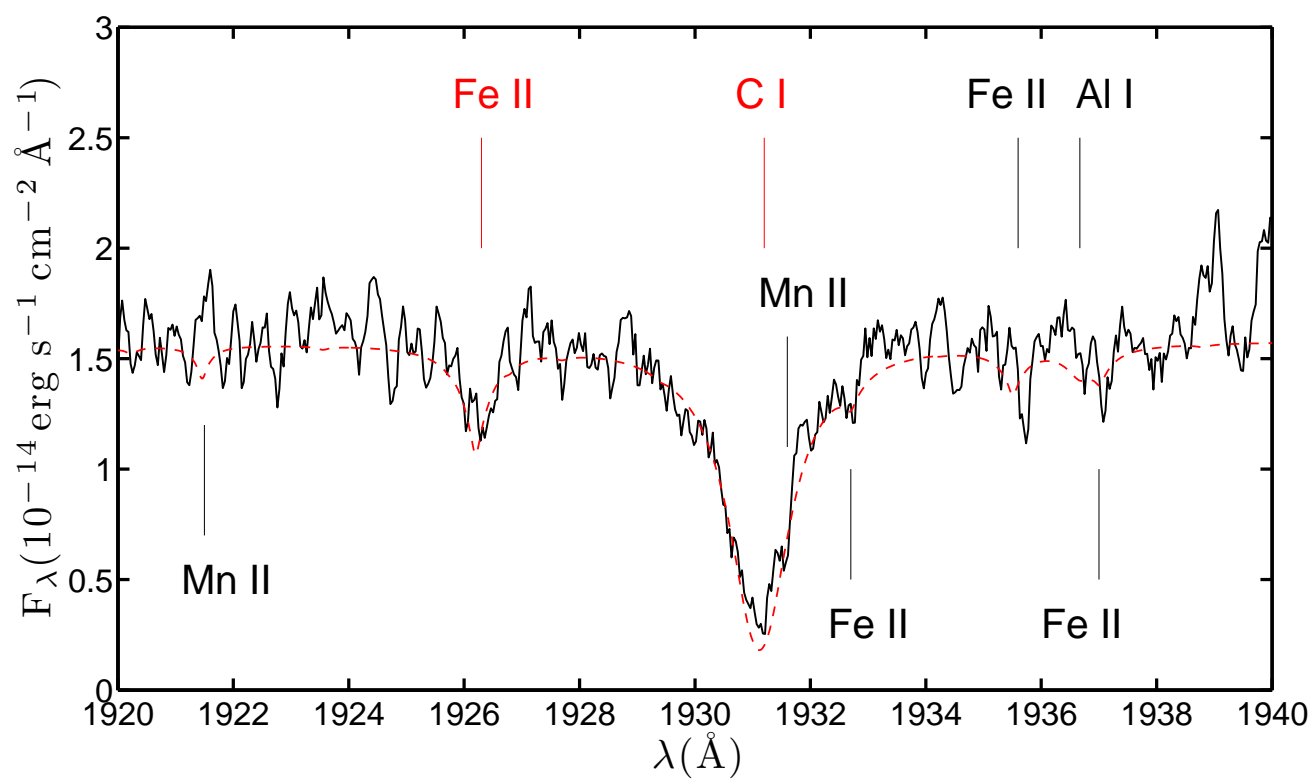

Fig. 2.- Similar to Figure 1 except for PG 1225-079 with abundances from Table 5. The data were smoothed with a 5 pixel boxcar. 
are upper limits.

\subsection{Magnesium and Zinc}

In PG 1225-079, Mg I 2026.4 $\AA$ and Zn II $2026.1 \AA$ are heavily blended. As shown in Figure 5, our best fit model which matches the measured EW of the absorption feature requires $[\mathrm{Mg}] /[\mathrm{He}]=-7.6$ and $[\mathrm{Zn}] /[\mathrm{He}]=-11.3$. These values are individually taken as upper limits due to the blending. However, the reported magnesium abundance is $-7.27 \pm$ 0.06 from the optical data (Klein et al. 2011), which is largely based on three Mg lines but the detections for two lines are only $2 \sigma$. Wolff et al. (2002) reported $[\mathrm{Mg}] /[\mathrm{He}]$ to be -7.6 \pm 0.6 from the IUE data. Averaging these measurements, our final magnesium abundance is $-7.50 \pm 0.20$. Because of the blending, the zinc abundance is only an upper limit. This provides the first stringent constraint on zinc in an extrasolar planetesimal.

\subsection{Silicon}

In PG 1225-079, we measured two silicon lines, Si II $1808.0 \AA$ and Si II $1816.9 \AA$, as shown in Figure 4. Si II $1808.8 \AA$ arises from the ground state and the photospheric line can be distorted by interstellar absorption. However, its measured EW is only $87 \pm 11 \mathrm{~m} \AA$ in $\zeta$ Oph, a star at a distance of $112 \mathrm{pc}$ with a large amount of foreground interstellar gas (Morton 1975). Considering PG 1225-079 is only 26 pc away, it has much less interstellar absorption. The measured $\mathrm{EW}$ is $936 \pm 109 \mathrm{~m} \AA$ and we conclude that Si II $1808.0 \AA$ is largely photospheric and essentially free from interstellar absorption. The shape of Si II 1808.0 $\AA$ in the model does not quite fit the data; but the measured EW of the data, which is key in the abundance determination, has a good agreement with that in the model. Using these two Si II lines, we derive a final silicon abundance of $-7.45 \pm 0.10$, in agreement with,

but much better than the reported IUE abundance of $-7.5 \pm 0.5$ (Wolff et al. 2002) and the previous optical upper limit of -7.27 (Klein et al. 2011).

\subsection{Iron}

In the COS data for PG 1225-079, there are six Fe II lines with EWs larger than 100 $\mathrm{m} \AA$. Four of them are shown in Figures 2 and 5 . We derived an iron abundance of -7.45 \pm 0.23 , in good agreement of the optical value of $-7.42 \pm 0.07$, which is based on 28 highSNR iron lines (Klein et al. 2011). Because the ultraviolet data are noisier, we adopt the 


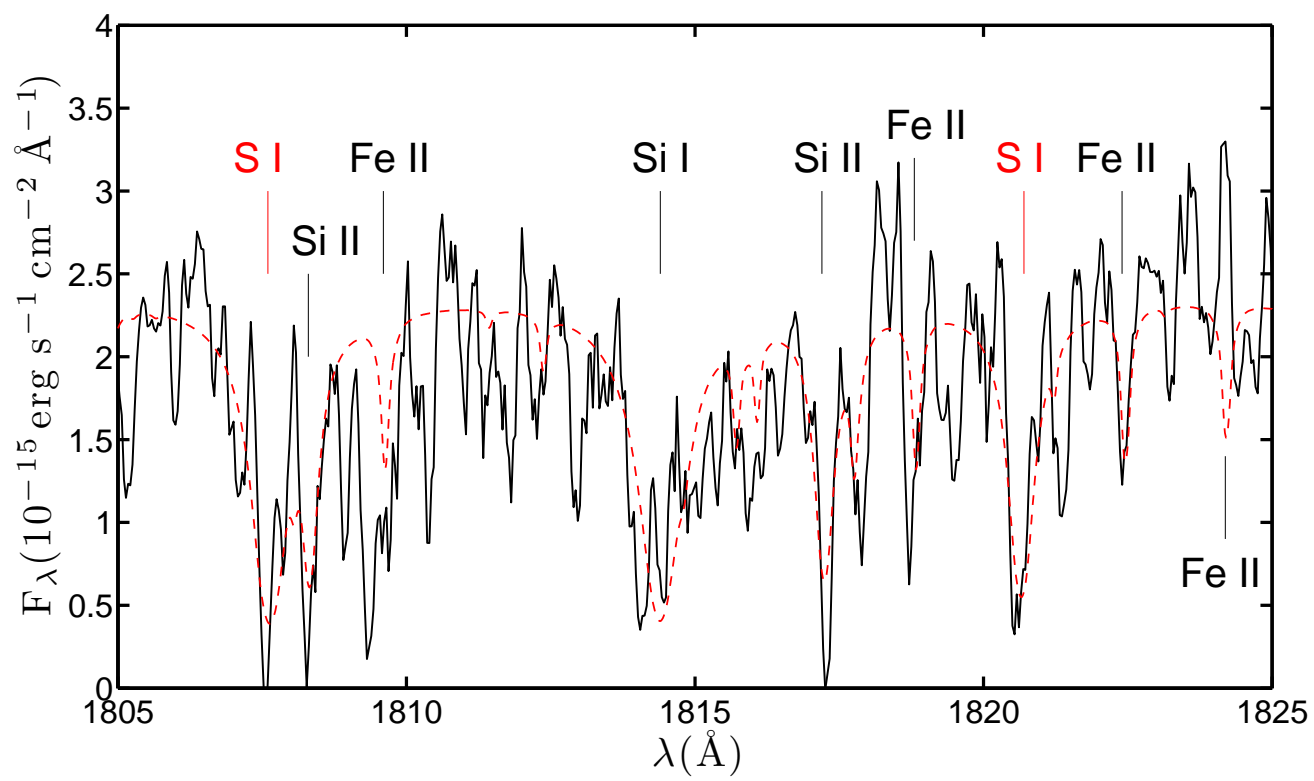

Fig. 3. - HST/COS spectrum of GD 362. All notations are the same as Figure 1 and the data are smoothed by a 5 pixel boxcar. S I $1807.3 \AA$ and $1820.3 \AA$ are used for constraining the sulfur abundance.

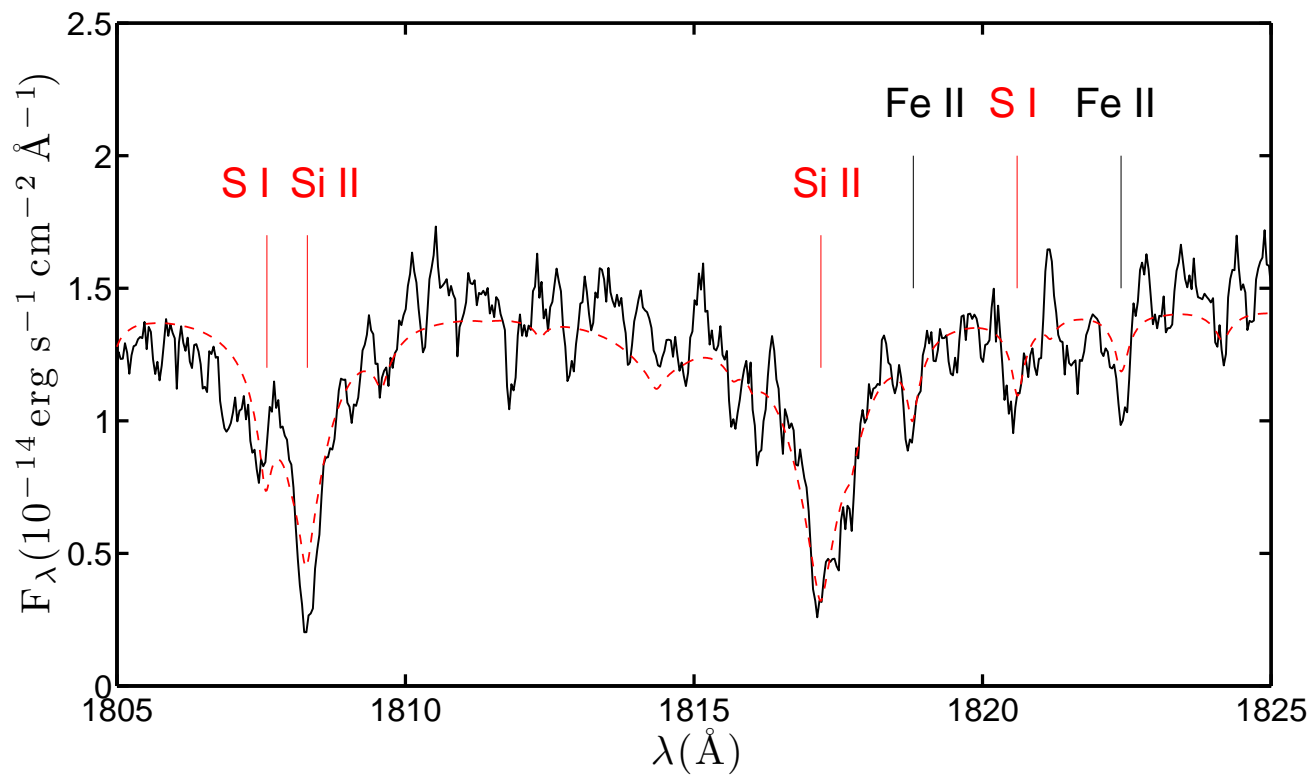

Fig. 4.- Similar to Figure 3 except for PG 1225-079. Si II $1808.0 \AA$ and $1816.9 \AA$ lines are used for determining the silicon abundance. 


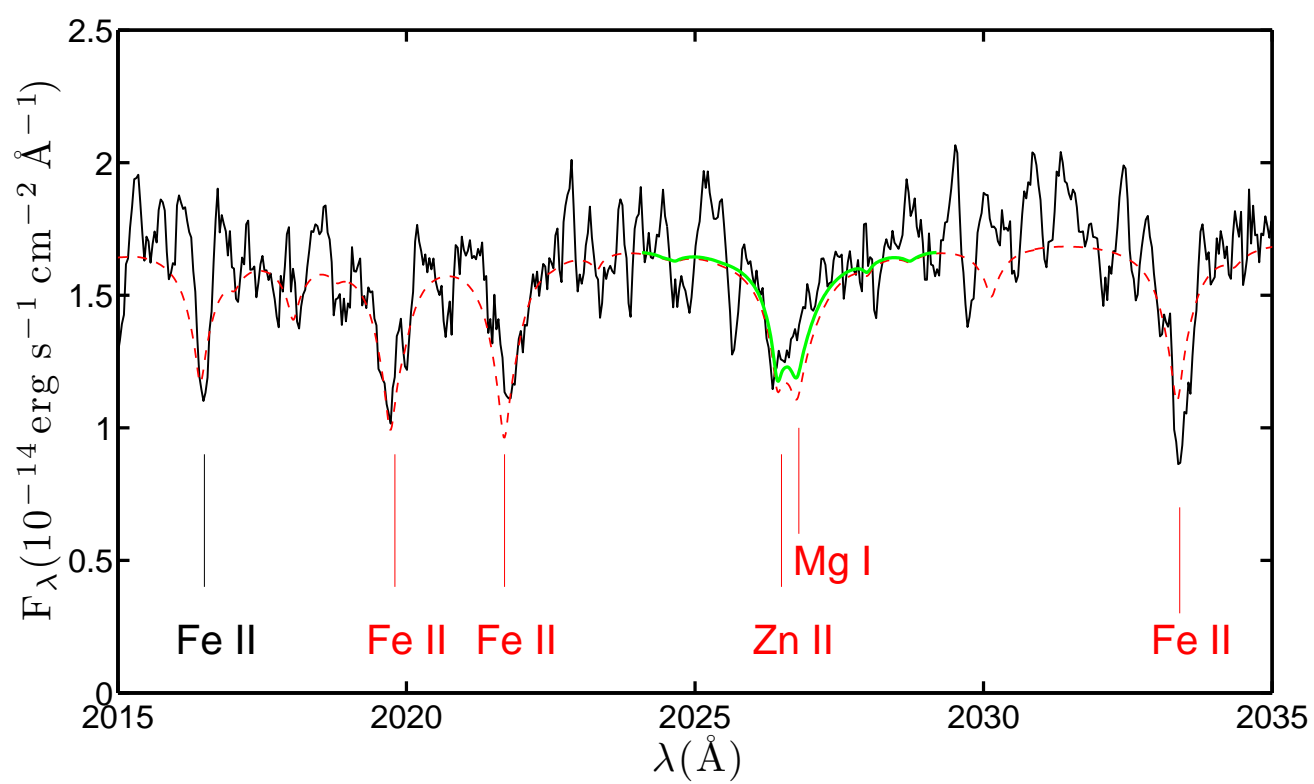

Fig. 5.- HST/COS spectrum of PG 1225-079 and the data are smoothed by a 5 pixel boxcar. All notations are the same as Figure 1. Zn II 2026.1 $\AA$ and Mg I 2026.5 $\AA$ are used for determining the $\mathrm{Zn}$ and $\mathrm{Mg}$ abundances, respectively. The green line presents the best fit model that matches the overall EW of the absorption feature at $2026 \AA([\mathrm{Mg}] /[\mathrm{He}]=$ $-7.6,[\mathrm{Zn}] /[\mathrm{He}]=-11.3)$; the red line is the adopted model combining with the optical results $([\mathrm{Mg}] /[\mathrm{He}]=-7.5,[\mathrm{Zn}] /[\mathrm{He}]=-11.3)$. Fe II 2019.4 $\AA, 2021.4 \AA, 2033.0 \AA$ and $1926.0 \AA$ in Figure 2 are used for determining the iron abundance.

optically-derived iron abundance.

\section{COMPARISON WITH SOLAR SYSTEM OBJECTS}

Combined with previous data, we now have determined the abundances of 16 elements heavier than helium in the atmosphere of GD 362 and 11 heavy elements in PG 1225-079. However, the measured composition need not be identical to the composition of the accreted planetesimal because different elements gravitationally settle at different rates in a white dwarf atmosphere. Three major phases are proposed for a single accretion event: build-up, steady-state and decay (Dupuis et al. 1993; Koester 2009).

Because an infrared excess is found for GD 362 and PG 1225-079 (Becklin et al. 2005; Kilic et al. 2005; Farihi et al. 2010), the accretion should be either in the build-up or steadystate phase. The timescale for build-up stage is comparable to the settling times (Koester 
2009); it is $\sim 10^{5} \mathrm{yr}$, for GD 362 and PG 1225-079 (see Tables 4 and 5). The rest of the disk-host stage should all be under the steady-state approximation. The dust disk lifetime has been under intensive studies for a few years but the values are still very uncertain, including $10^{5}$ yr (Farihi et al. 2009; Rafikov 2011b), $10^{6}$ yr (Rafikov 2011a; Girven et al. 2012; Farihi et al. 2012) and up to $10^{7}$ yr (Barber et al. 2012). The true disk lifetime might have a range but it is likely to be longer than the settling times. Furthermore, Zuckerman et al. (2010) suggested that steady-state approximation is the dominant situation for white dwarf accretion event based on a study of helium dominated stars; the settling times are only $0.1 \%$ of their cooling times but $30 \%$ of them show atmospheric pollution. GD 362 and PG 1225-079 are more likely to be under the steady-state approximation and that is the main focus of this paper.

In the steady-state model, the observed concentration of an element is dependent on the time it takes to sink out of the convective envelope. To derive the theoretical settling times and obtain an improved understanding of the uncertainties, we formulated several numerical experiments with the code for the envelope structure and corrected two errors found in our previous calculations of diffusion timescales. In the course of changing the equations describing element diffusion from the version in Paquette et al. (1986) (Equation 4) to the one in Pelletier et al. (1986) (Equation 5), which is more accurate in the case of electron degeneracy, one of us (D.K.) discovered an error in the former paper. A factor of $\rho^{1 / 3}$ is missing in the second alternative of Equation 21, which we had not noticed before. A rederivation of all our equations uncovered another error in our implementation of the contribution of thermal diffusion. These errors have only a very small effect in stars with relatively shallow convection zones, like the hydrogen-dominated white dwarfs. However, for helium-dominated white dwarfs with $\mathrm{T}<15,000 \mathrm{~K}$ and a deep convection zone, the diffusion timescales can be slower by factors 2-3 relative to our earlier calculations 5 . The accretion rate $\dot{M}\left(Z_{i}\right)$ of an element $\mathrm{Z}$ is calculated as (Koester 2009)

$$
\dot{M}\left(Z_{i}\right)=\frac{M_{c v z} X\left(Z_{i}\right)}{t_{\text {set }}\left(Z_{i}\right)}
$$

where $\mathrm{M}_{c v z}$ is the mass of the convective envelope. $\mathrm{X}\left(\mathrm{Z}_{i}\right)$ is the mass fraction of the element $\mathrm{Z}_{i}$ relative to the dominant element in the atmosphere, either hydrogen or helium; $t_{\text {set }}\left(\mathrm{Z}_{i}\right)$ is the settling time. A longer settling time corresponds to a lower diffusion flux. Fortunately, the relative timescales for different elements, which are important for the determination of the abundances in the accreted matter, change much less.

\footnotetext{
${ }^{5}$ Updated diffusion timescales can be obtained at http://www.astrophysik.uni-kiel.de/ koester/astrophysics/
} 
For GD 362 and PG 1225-079, compared to previously published values, the settling times listed in Tables 4 and 5 typically increase by factors of 2-3 while the mass of the convective zone is 0.13 dex smaller for GD 362 and 0.05 dex larger for PG 1225-079 (Table 3). These corrections lead to smaller total accretion rates by a factor of 3 for both stars.

The next step is to compare the composition of the accreted parent body with those of solar system objects. We choose the summed mass of all the major elements as the normalization factor so that the analysis is independent of the chemical property and abundance uncertainty of each individual element. However, one complication is that no oxygen lines are detected in either GD 362 or PG 1225-079 due to their low photospheric temperatures relative to other helium-dominated white dwarfs; only upper limits were obtained for this major element. Therefore, our approach is to compare the mass fraction of an element relative to the summed mass of the common elements Mg, Si and Fe. For solar system objects, we include 80 representative and well-analyzed meteorite samples mostly from Nittler et al. (2004). We also include the bulk composition of Earth from Allègre et al. (2001) and an updated carbon abundance from Marty (2012). For our purpose, Earth appears to be chondritic and its bulk composition approaches CV chondrites even though Earth has experienced some post-nebular processing, such as differentiation and collisions.

\subsection{GD 362: Accretion from a Mesosiderite Analog?}

In Figure 6, we compare the abundances of all 18 elements, including upper limits, of the accreted material in GD 362 with CI chondrites, which are the most primitive material in the solar system. The composition of CI chondrites is almost identical to the solar photosphere, with the exception of depletion of volatile elements $\mathrm{C}, \mathrm{N}$ as well as $\mathrm{H}$ and noble gases. The parent body accreted onto GD 362 looks nothing like a CI chondrite, as first pointed out in Zuckerman et al. (2007). For the volatile elements, the mass fraction of $\mathrm{C}$ and $\mathrm{S}$ are depleted by at least a factor of 7 and 3, respectively, relative to CI chondrites; refractory elements, such as V, Ca, Ti and Al, are all enhanced.

Though oxygen is not detected in GD 362, its stringent upper limit can still provide

useful insights. Following Klein et al. (2010), we can calculate the required number of oxygen atoms to form oxides $\mathrm{Z}_{p(Z)} \mathrm{O}_{q(Z)}$ as

$$
n(O)=\sum_{Z} \frac{q(Z)}{p(Z)} n(Z)
$$

Hydrogen is excluded here because GD 362 has an enormous amount and it might not be associated with the parent body or bodies currently in its atmosphere (see Appendix A). 


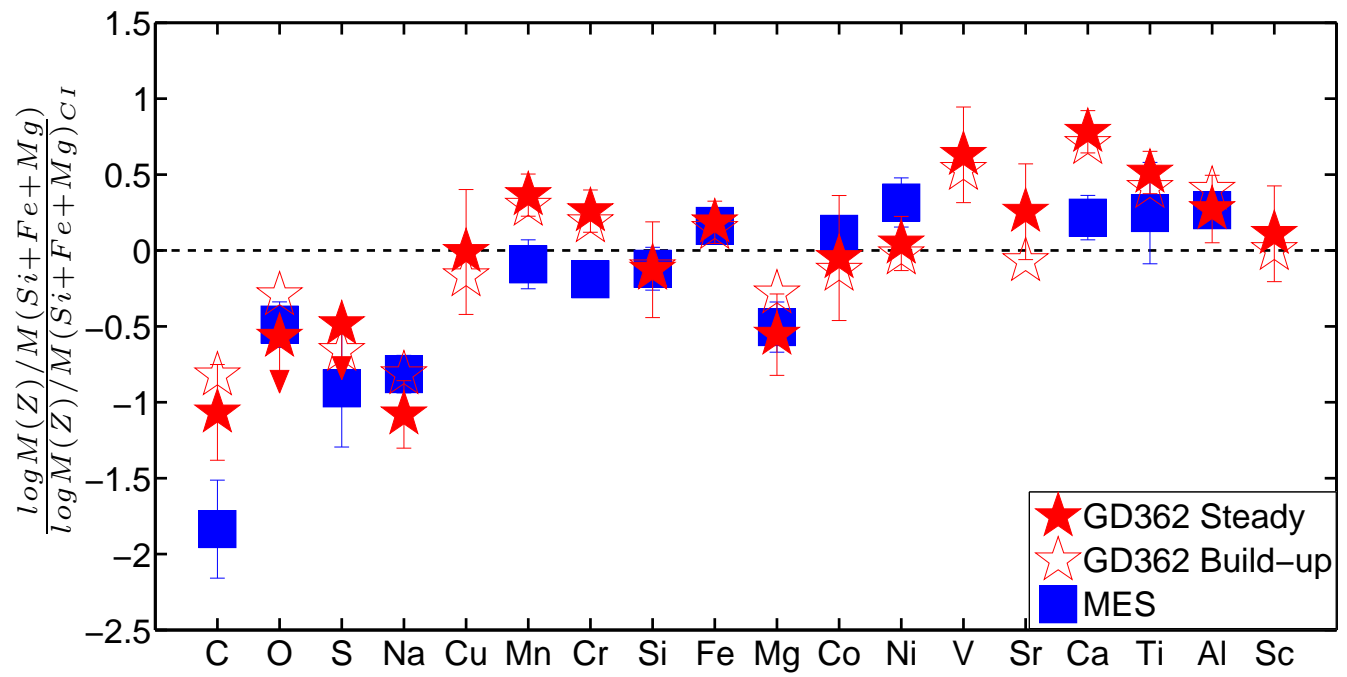

Fig. 6.- Mass fractions of heavy elements in GD 362 with respect to the summed mass of silicon, iron and magnesium from Table 4. The abundances are normalized to those of CI chondrites. The elements are ordered by decreasing volatility. The filled stars represent the steady-state approximation while the open stars are in the build-up stage. $1 \sigma$ error bars and arrows for upper limits are plotted only for the steady-state approximation for clarity. We also plot the average values for five mesosiderites (Emergy, Barea, Patwar, Dyarrl Island and ALH 77219) from Nittler et al. (2004); $1 \sigma$ deviations are shown as blue error bars. The error bars are smaller than the symbol when they are not visible in the figure. There are no reported whole rock fractions of $\mathrm{Cu}, \mathrm{V}, \mathrm{Sr}$ and $\mathrm{Sc}$ in mesosiderites.

Under the steady-state approximation, $[\mathrm{O}] /[\mathrm{He}]=-5.07$ is required to form $\mathrm{MgO}, \mathrm{Al}_{2} \mathrm{O}_{3}$, $\mathrm{SiO}_{2}$ and $\mathrm{CaO}$; this value is comparable to the observed oxygen upper limit of -5.14 . However, Fe is the most abundant heavy element in the atmosphere of GD 362 and there is insufficient oxygen to tie it up in either $\mathrm{FeO}$ or $\mathrm{Fe}_{2} \mathrm{O}_{3}$. Thus, most, if not all the iron in the parent body is in metallic form, which is very different from CI chondrites where most iron is in oxides (Nittler et al. 2004).

O'Neill \& Palme (2008) suggested that $[\mathrm{Mn}] /[\mathrm{Na}]$ can be used as an indicator of postnebular processing. For example, $[\mathrm{Mn}] /[\mathrm{Na}]$ is -0.79 for all chondrites as well as the solar photosphere while non-chondritic objects have a much higher value. Interestingly, $[\mathrm{Mn}] /[\mathrm{Na}]$ is $0.65 \pm 0.22$ for GD 362, which is larger than -0.01 for Mars and 0.32 for the Moon (O'Neill \& Palme 2008). This suggests that the planetesimal accreted onto GD 362 is likely to be non-chondritic and have experienced some post-nebular processing. Zuckerman et al. (2007) compared the $[\mathrm{Na}] /[\mathrm{Ca}]$ ratio in GD 362 with solar system objects and reached a 
similar conclusion; the accreted planetesimal was non-chonridtic. The only other polluted white dwarf with both $\mathrm{Mn}$ and Na detections is WD J0738+1835 wherein $[\mathrm{Mn}] /[\mathrm{Na}]=-0.54$ \pm 0.19 (Dufour et al. 2012); this agrees with the chondritic value within the uncertainties.

To find the best solar system analog to the parent body accreted onto GD 362, we calculated a reduced chi-squared value for each object in our sample $\left(\chi_{r e d}^{2}\right)$, defined as:

$$
\chi_{\text {red }}^{2}=\frac{1}{N} \sum_{i=1}^{N} \frac{\left(M_{w d}\left(Z_{i}\right)-M_{m t r}\left(Z_{i}\right)\right)^{2}}{\sigma_{w d}^{2}\left(Z_{i}\right)}
$$

where $\mathrm{N}$ is the total number of elements considered in the analysis. $\mathrm{M}_{w d}\left(\mathrm{Z}_{i}\right)$ and $\mathrm{M}_{m t r}\left(\mathrm{Z}_{i}\right)$ represent the mass fraction of an element $Z_{i}$ relative to the summed mass of $\mathrm{Mg}, \mathrm{Si}$ and $\mathrm{Fe}$ in the extrasolar planetesimal and solar system objects, respectively. $\sigma_{w d}\left(\mathrm{Z}_{i}\right)$ is the propagated uncertainty in mass fraction.

For GD 362, we calculated $\chi_{\text {red }}^{2}$ for 11 heavy elements, C, Na, Mg, Al, Si, Ca, Ti, Cr, $\mathrm{Mn}, \mathrm{Fe}$ and $\mathrm{Ni}$, which have detections both in GD 362 and the meteorite sample6. The results are shown in Figure 7 for both steady-state and build-up approximations. There is no qualitative difference between these two models and mesosiderites provide the best fit considering all 11 elements. In particular, the mesosiderite ALH 77219 can match the overall abundance pattern to $95 \%$ confidence level. As shown in Figure 6, the abundance of individual elements agrees within $2 \sigma$ between mesosiderites and the planetesimal accreted onto GD 362.

Mesosiderites are a rare type of stoney-iron meteorite with equal amounts of silicates and metallic iron and nickel. One mystery about mesosiderties is that the Si-rich crust and Fe, Ni-rich core materials are abundant but the olivine Mg-rich mantle seems to be missing. One model for the formation of mesosiderites is that a $200-400 \mathrm{~km}$ diameter asteroid with a molten core was nearly catastrophically disrupted by a $50-150 \mathrm{~km}$ diameter projectile at 4.42-4.52 Gyr ago (Scott et al. 2001). The collision mixed the target's molten core with its crustal material but excluded the large and hot mantle fragments. The planetesimal accreted onto GD 362 may have been formed in a similar way.

While mesosiderites may be a prototype for the accreted planetesimal onto GD 362, there are three major hurdles for this hypothesis to overcome. First, in the model of Scott et al. (2001), only half of the original mass of a 200-400 km diameter asteroid was maintained after the collision and the final product only contains about $10 \%$ mesosiderite-like material

\footnotetext{
${ }^{6}$ For a couple of meteorites with no reported carbon abundance, we compute the $\chi_{\text {red }}^{2}$ for the other 10 elements.
} 


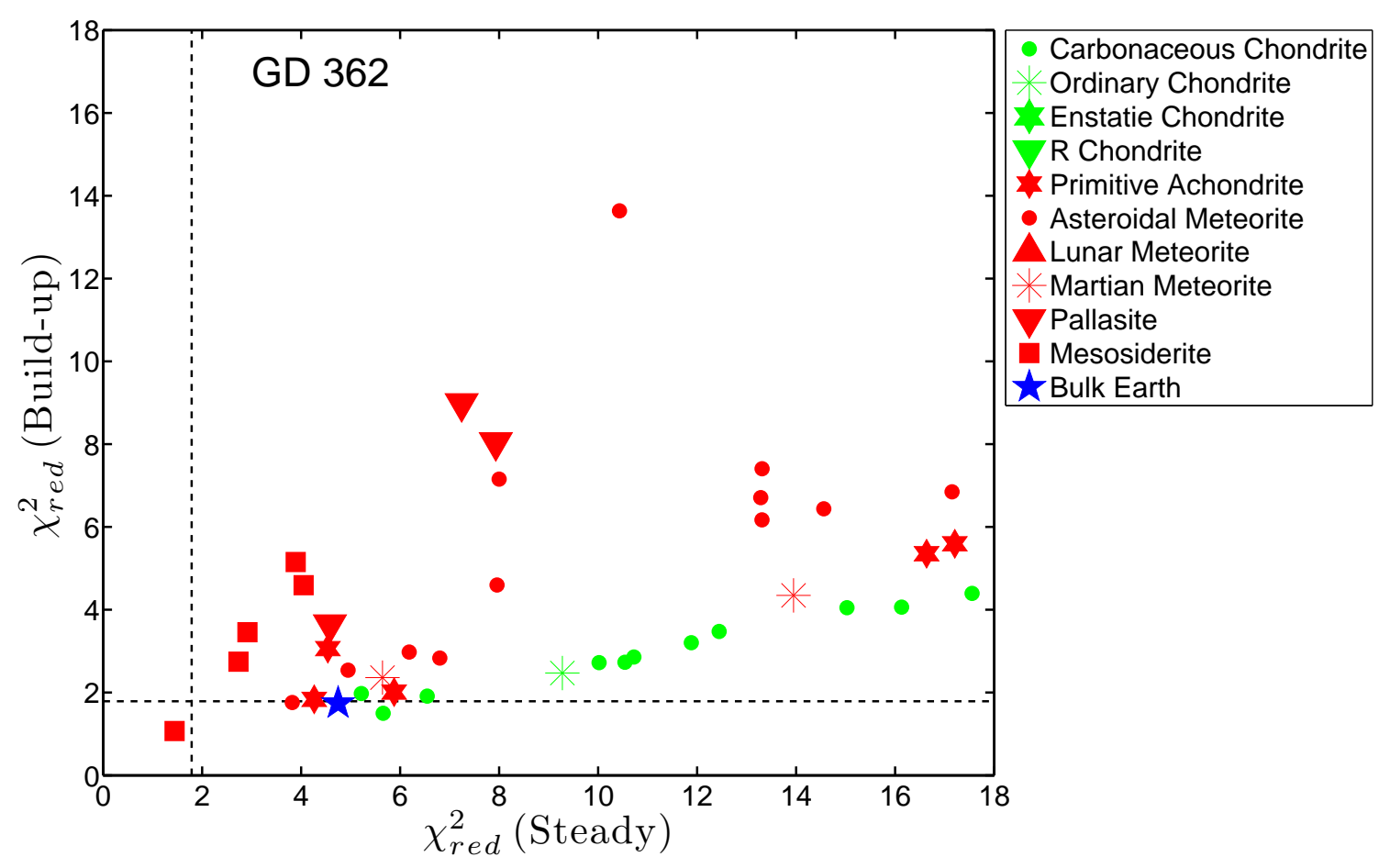

Fig. 7.- This figure shows $\chi_{\text {red }}^{2}$ values defined in Equation (3), which compares the abundances of $\mathrm{C}, \mathrm{Na}, \mathrm{Mg}, \mathrm{Al}, \mathrm{Si}, \mathrm{Ca}, \mathrm{Ti}, \mathrm{Cr}, \mathrm{Mn}, \mathrm{Fe}$ and $\mathrm{Ni}$ in the accreted material in GD 362 with 80 meteorites and bulk Earth. The x-axis denotes $\chi_{\text {red }}^{2}$ calculated for the composition under the steady-state approximation while the y-axis is for the build-up phase. The dashed lines represent $95 \%$ confidence level. There are a couple of meteorites with a large $\chi_{\text {red }}^{2}$ and they are not shown in the current scale. There is no qualitative difference between the steady-state and build-up models. Mesosiderites, particuarly ALH 77219, provide the best fit to the abundance pattern observed in GD 362. Notes: "Chondritic" materials include: (i) carbonaceous chondrites: CI, CK, CM, CO, CR and CV; (ii) ordinary chondrites: H, L and LL; (iii) enstatite chondrites: EH and EL; (iv) R chondrite. "Non-chondritic" materials consist of achondrites and stoney-iron meteorites. No iron meteorite is included in our analysis because their bulk composition is dominated by $\mathrm{Fe}$ and $\mathrm{Ni}$, with very few trace elements reported. Achondrites include: (i) primitive achondrites: acapulcoites, lodranites, winonaites and ureilites; (ii) asteroidal meteorites: angrites, aubrites, brachinites and howardite-eucrite-diogenite; (iii) Martian meteorites: shergottie, Nakhlites and Chassignites; (iv) Lunar meteorites. For stoney-iron meteorites, we include (i) mesosiderites; (ii) pallasites. Most of the meteorite data are from Nittler et al. (2004) and the compositions for some Martian meteorites are from McSween (1985). The bulk composition of Earth is from Allègre et al. (2001) and the carbon abundance is from a more recent study of Marty (2012), which is a factor of 3 lower than the lower limit reported in Allègre et al. (2001). 
by mass. This is equivalent to a $75-150 \mathrm{~km}$ diameter object. Mesosiderites that fall on Earth are only small fragments and the $180 \mathrm{~kg}$ NWA 2924 is among the largest (Meteorite Bulletin Databas 7 ). However, the parent body accreted onto GD 362 has a minimum mass of 2.7 $\times 10^{22} \mathrm{~g}, \sim 260 \mathrm{~km}$ in diameter for an assumed density of $3 \mathrm{~g} \mathrm{~cm}^{-3}$. It is unclear whether the same kind of collision can produce a mesosiderite parent body this big. Second, the mass fraction of hydrogen in mesosiderites is less than $0.2 \%$; it cannot explain how there is $5 \times 10^{24} \mathrm{~g}$ hydrogen in the atmosphere of GD 362. Possibly, hydrogen was accreted during earlier events and it has been atop the atmosphere ever since (see Appendix A for more discussion). Third, GD 362 is currently accreting from its circumstellar disk and the disk material should also resemble the composition of mesosiderites. However, the shape of the mid-infrared spectrum for mesosiderite, which is dominated by a sharp peak at $9.13 \mu \mathrm{m}$ and several other bands at $10.6 \mu \mathrm{m}$ and $11.3 \mu \mathrm{m}$ (Morlok et al. 2012), cannot fully account for the broad $10 \mu \mathrm{m}$ silicate emission feature observed for GD 362 (Jura et al. 2007). This does not completely exclude the mesosiderite hypothesis but emission from some additional material is required to fully reproduce the observed infrared spectrum for GD 362. Mesosiderites are a good candidate for the parent body accreted onto GD 362 but there are remaining unresolved issues.

\subsection{PG 1225-079: Accretion from a Planetesimal with No Single Solar System Analog}

In Figure 8, we show a comparison of the mass fractions of 16 elements, including upper limits between PG 1225-079 and CI chondrites. Though the carbon abundance is approaching the chondritic value, the accreted planetesimal differs a lot from CI chondrites; the mass fraction of $\mathrm{S}$ is depleted by at least a factor of 40 while $\mathrm{Zn}$ is depleted by at least a factor of 8. In contrast, refractories, such as $\mathrm{V}, \mathrm{Ca}, \mathrm{Ti}$ and $\mathrm{Sc}$ are all enhanced. The overall pattern of relatively high carbon abundance and enhanced mass fractions of refractory elements does not follow a single condensation sequence and post-nebular processing is required.

As shown in Figure 9, PG 1225-079 has a [C]/[S] value that is no smaller than the solar ratio, which is very different from other polluted white dwarfs and meteorites. Carbon and sulfur are among the most volatile elements that we can measure and their $50 \%$ condensation temperatures are $40 \mathrm{~K}$ and $655 \mathrm{~K}$, respectively (Lodders 2003). Most of the meteorites as well as polluted white dwarfs have a $[\mathrm{C}] /[\mathrm{S}]$ ratio lower than the solar value, which can be explained by condensation at a temperature between 40 and $665 \mathrm{~K}$ though this is not

\footnotetext{
${ }^{7}$ http://www.lpi.usra.edu/meteor/
} 


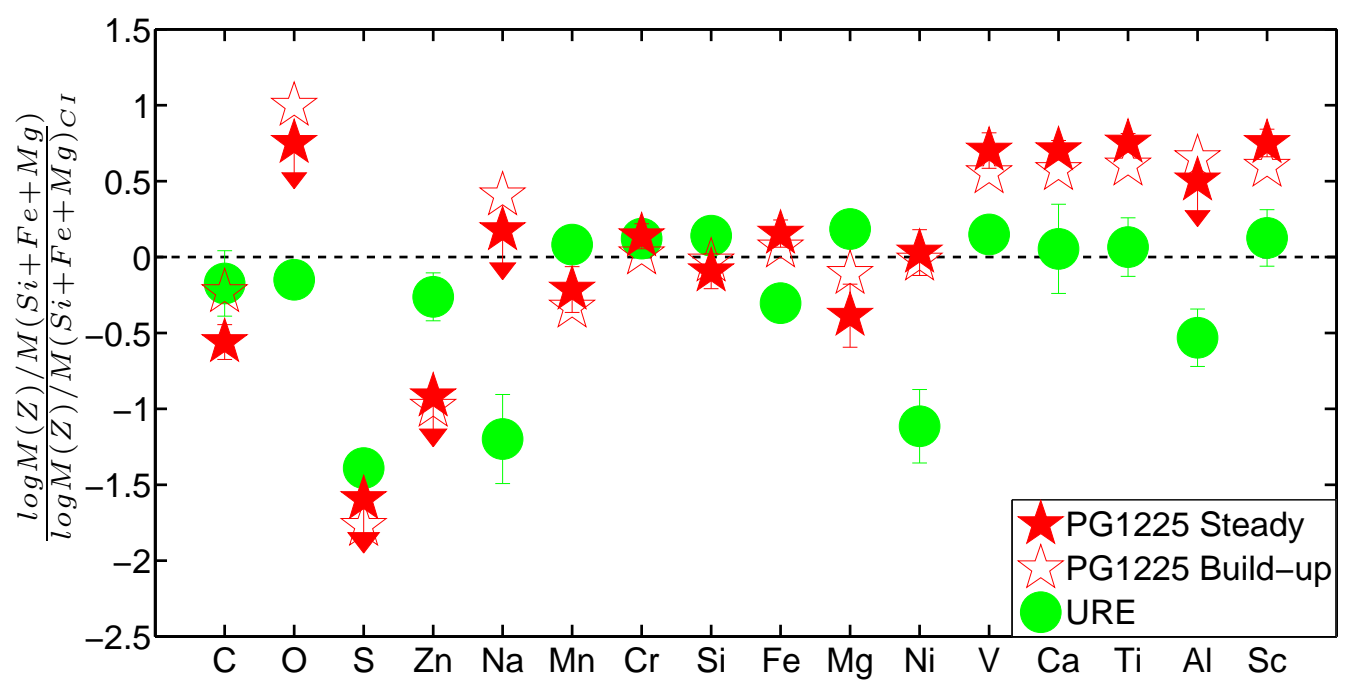

(a)

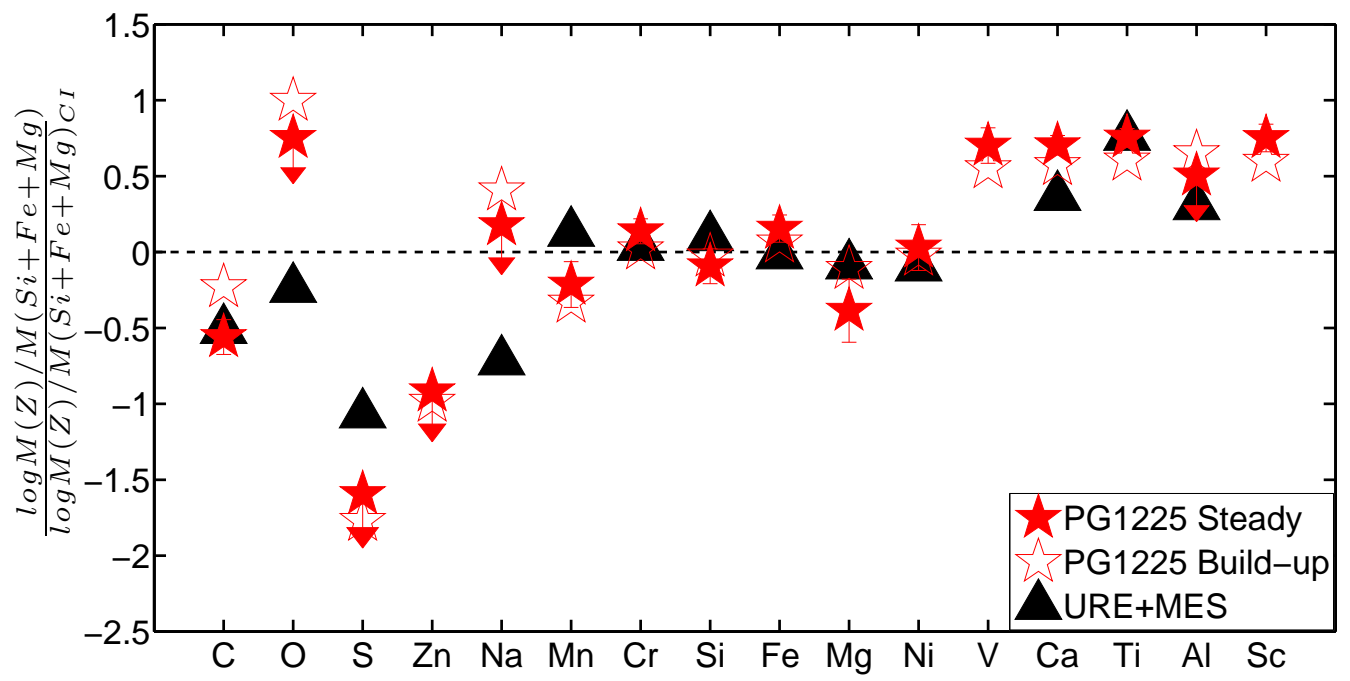

(b)

Fig. 8.- (a) Similar to Figure 6] except for PG 1225-079 with the abundances in Table 5 . Ureilites can match the high carbon low sulfur pattern in PG 1225-079 but fail with the other elements. The abundances for ureilites are from Warren et al. (2006). (b) The best-fit model for the composition in PG 1225-069 under the steady-state approximation is 30\% by mass ureilite North Haig and 70\% mesosiderite Dyarrl Island. There are no reported bulk compositions of Zn, V or Sc for the North Haig and Dyarrl Island meteorites. 
necessarily true for all of them. The only solar system analog to PG 1225-079 with similar high carbon, low sulfur pattern is ureilites, a type of primitive achondrites. Ureilites are the second largest achondrite group and it is suggested that its high carbon abundance is derived from a carbon-rich parent body, but the exact formation mechanism is not well understood Goodrich 1992). However, as can be seen in Figure 8(a), ureilites fail to match the overall composition of the parent body accreted onto PG 1225-079.

We performed a $\chi_{\text {red }}^{2}$ analysis between solar system objects and the accreted planetesimal in PG 1225-079, comparing 9 elements, C, Mg, Si, Ca, Ti, Cr, Mn, Fe and Nis. The result is shown in Figure 10. There is no single solar system object that can match all nine elements; the closest is carbonaceous chondrite. Regardless, as shown in Figure 8, the accreted abundance in PG 1225-079 is not at all identical to CI chondrites.

The infrared excess around PG 1225-079 corresponds to $\sim 500 \mathrm{~K}$ dust (Farihi et al. 2010); so far, only two white dwarfs are known to have such cool dust. The other 28 known disk-host stars all have $\sim 1000 \mathrm{~K}$ dust (Xu \& Jura 2012). One hypothesis is that the inner disk region was recently impacted by another asteroid and all the material was dissipated (Farihi et al. 2010; Jura 2008). If that is the case, PG 1225-079 can be accreting from a blend of two planetesimals, rather than one single parent body. After testing different combinations of the 80 meteorites in our database, the best fit model to the steady-state approximation consists of 30\% ureilite North Haig and 70\% mesosiderite Dyarrl Island by mass. This blend is also marked in Figure 10. Detailed abundance comparison is shown in Figure 8(b); the abundances of $\mathrm{S}, \mathrm{Mn}$ and $\mathrm{Ca}$ do not agree as well as the other elements but are all within $2 \sigma$. A possible scenario is that one extrasolar ureilite (mesosiderite) analog first got tidally disrupted and more recently, another mesosiderite (ureilite) analog impacted the disk and was blended with the previous material.

\section{ASSESSING THE FORMATION MECHANISMS OF EXTRASOLAR PLANETESIMALS}

Having established that the parent bodies accreted onto GD 362 and PG 1225-079 are beyond primitive, we now extend our analysis to other extrasolar planetesimals. We are most interested in understanding the formation mechanisms of extrasolar planetesimals and whether these are dominated by nebular or post-nebular processing. Jura \& Xu (2013) suggested collisional rearrangement is important in determining the final composition of

\footnotetext{
${ }^{8}$ Similar to the case of GD 362, for the meteorites with no reported carbon abundance, we only calculated $\chi_{\text {red }}^{2}$ for the other 8 elements.
} 


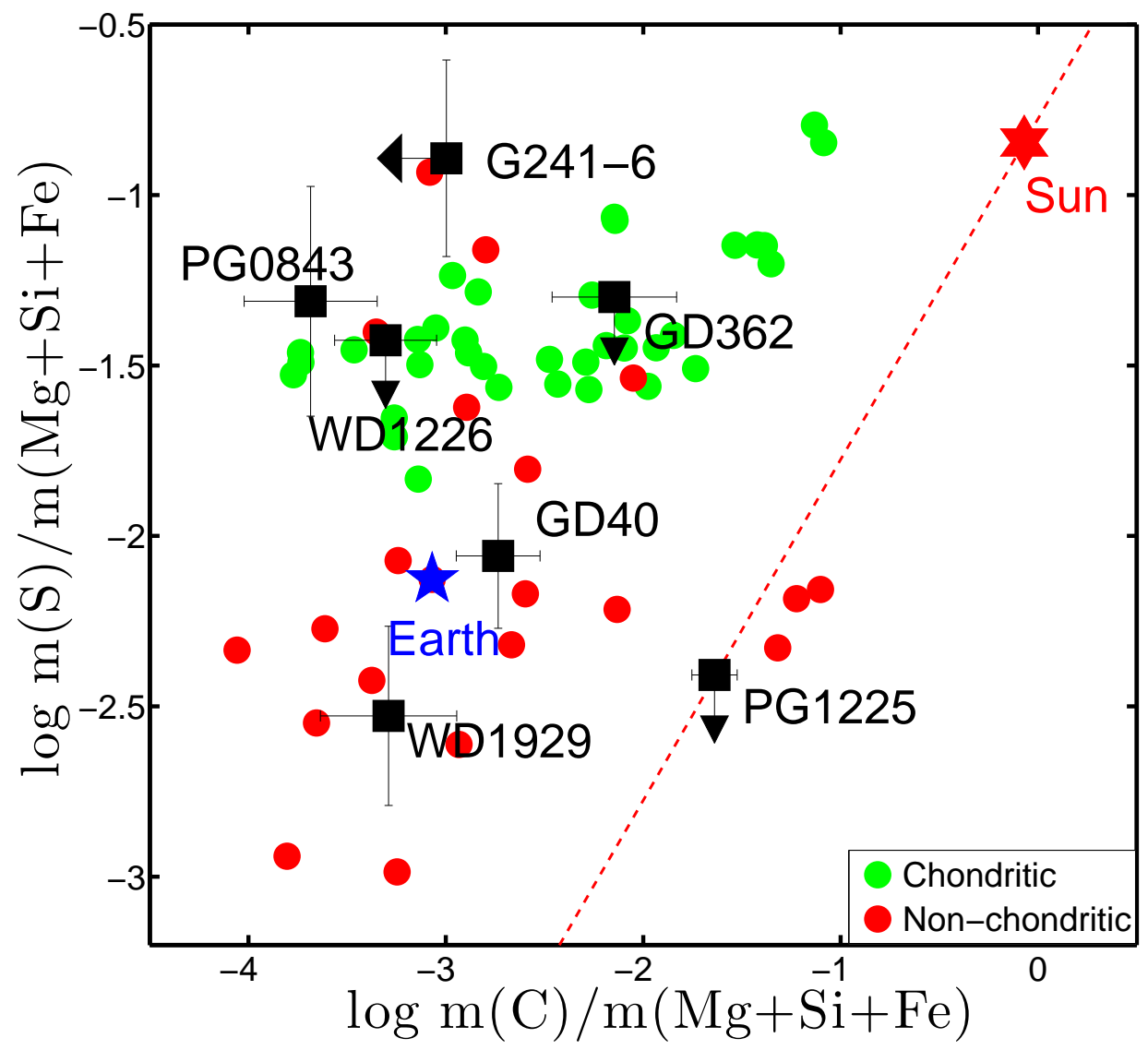

Fig. 9.- Mass fraction of $\mathrm{S}$ and $\mathrm{C}$ over the sum of Fe, $\mathrm{Mg}$ and Si for solar system objects as well as polluted white dwarfs with positive detection of carbon or sulfur or both. Due to the presence of an infrared excess except for G241-6 (see discussion in Appendix B), all white dwarfs are plotted under the steady-state approximation . $1 \sigma$ uncertainties are plotted. The red dashed line denotes constant solar $[\mathrm{C}] /[\mathrm{S}]$. Most meteorites have a lower $[\mathrm{C}] /[\mathrm{S}]$ than the solar value. However, $[\mathrm{C}] /[\mathrm{S}]$ in $\mathrm{PG} 1225-079$ is no smaller than solar and the closest solar system analog is ureilites. References: WD 1929+012, PG 0843+517, WD 1226+110: Gänsicke et al. (2012); GD 40, G241-6: Jura et al. (2012); GD 362 and PG 1225-079: this paper; Solar abundance: Lodders (2003); for solar system objects, the references are listed in the caption of Figure 7 


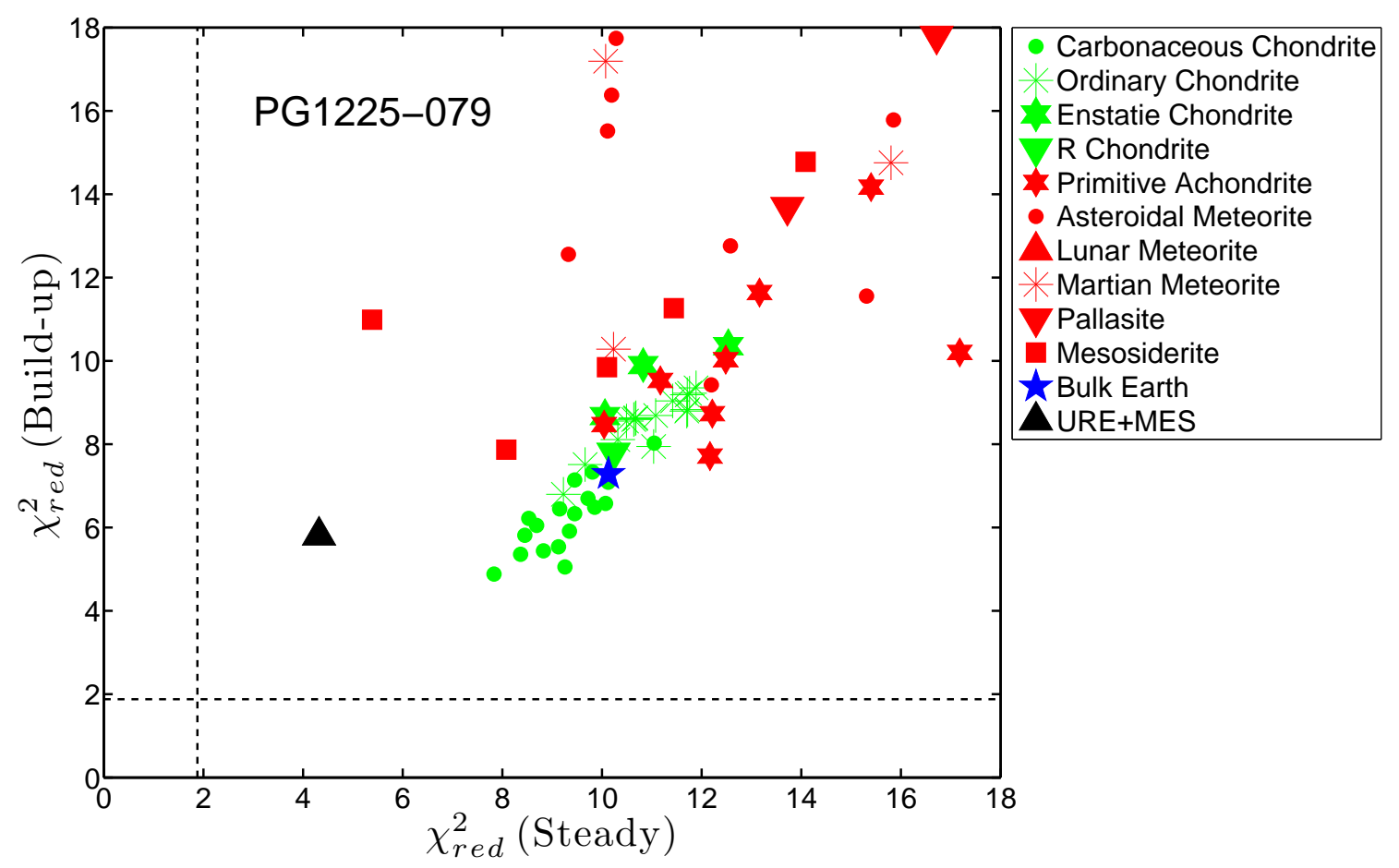

Fig. 10.- Similar to Figure 7 except for PG 1225-079 comparing 9 elements, C, Mg, Si, $\mathrm{Ca}, \mathrm{Ti}, \mathrm{Cr}, \mathrm{Mn}, \mathrm{Fe}$ and $\mathrm{Ni}$. We see that no solar system object comes close to the overall abundance pattern in PG 1225-079. The black triangle represents $30 \%$ by mass ureilite and $70 \%$ mesosiderite as shown in Figure 8(b), which is the best match for the composition of PG 1225-079 in the steady-state approximation.

extrasolar planetesimals based on the scatter in $[\mathrm{Mg}] /[\mathrm{Ca}]$ ratios in 60 externally-polluted white dwarfs. Here, we compile a sample of well-studied externally-polluted white dwarfs with abundance determinations of at least 9 elements. There are 9 stars in total, as listed in Table 6] and now we assess the formation mechanism for individual objects.

GD 40: As discussed in Jura et al. (2012) and Appendix B, the overall abundance pattern in GD 40 matches with carbonaceous chondrites and bulk Earth. Nebular condensation is sufficient to explain its observed composition.

WD J0738+1835: Dufour et al. (2012) found that there is a correlation between the abundance of an element and its condensation temperature: refractory elements are depleted while volatile elements are enhanced compared to bulk Earth. This indicates that the accreted planetesimal might be formed in a low temperature environment under nebular condensation. 
Table 6: Summary of Planetesimal Formation Mechanisms in 9 Well-Studied White Dwarfs

\begin{tabular}{|c|c|c|c|c|c|c|c|}
\hline star & Dom. & Dust & Volatile & Intermediate & Refractory & Process & Ref \\
\hline GD 40 & $\mathrm{He}$ & $\mathrm{Y}$ & C,S:,O,Mn, P & $\mathrm{Cr}, \mathrm{Si}, \mathrm{Fe}, \mathrm{Mg}, \mathrm{Ni}$ & $\mathrm{Ca}, \mathrm{Ti}, \mathrm{Al}$ & primitive & 1,2 \\
\hline WD J0738+1835 & $\mathrm{He}$ & $\mathrm{Y}$ & $\mathrm{O}, \mathrm{Na}, \mathrm{Mn}$ & $\mathrm{Cr}, \mathrm{Si}, \mathrm{Fe}, \mathrm{Mg}, \mathrm{Co}, \mathrm{Ni}$ & $\mathrm{V}, \mathrm{Ca}, \mathrm{Ti}, \mathrm{Al}, \mathrm{Sc}$ & primitive & 3,4 \\
\hline PG $0843+517$ & $\mathrm{H}$ & $\mathrm{Y}$ & $\mathrm{C}, \mathrm{S}, \mathrm{O}, \mathrm{P}$ & $\mathrm{Cr}, \mathrm{Si}, \mathrm{Fe}, \mathrm{Mg}, \mathrm{Ni}$ & $\mathrm{Al}$ & beyond-primitive(?) & 5 \\
\hline PG 1225-079 & $\mathrm{He}$ & $\mathrm{Y}$ & $\mathrm{C}, \mathrm{Mn}$ & $\mathrm{Cr}, \mathrm{Si}, \mathrm{Fe}, \mathrm{Mg}, \mathrm{Ni}$ & V,Ca,Ti,Sc & beyond-primitive & 6,7 \\
\hline NLTT 43806 & $\mathrm{H}$ & $\mathrm{N}$ & $\mathrm{Na}$ & $\mathrm{Cr}, \mathrm{Si}, \mathrm{Fe}, \mathrm{Mg}, \mathrm{Ni}$ & $\mathrm{Ca}, \mathrm{Ti}, \mathrm{Al}$ & beyond-primitive & 8 \\
\hline GD 362 & $\mathrm{He}$ & $\mathrm{Y}$ & $\mathrm{C}, \mathrm{Na}, \mathrm{Cu}, \mathrm{Mn}$ & $\mathrm{Cr}, \mathrm{Si}, \mathrm{Fe}, \mathrm{Mg}, \mathrm{Co}, \mathrm{Ni}$ & V,Sr,Ca,Ti,Al,Sc & beyond-primitive & 7,9 \\
\hline WD 1929+012 & $\mathrm{H}$ & $\mathrm{Y}$ & $\mathrm{C}, \mathrm{S}, \mathrm{O}, \mathrm{Mn}, \mathrm{P}$ & $\mathrm{Cr}, \mathrm{Si}, \mathrm{Fe}, \mathrm{Mg}, \mathrm{Ni}$ & $\mathrm{Ca}, \mathrm{Al}$ & $? ? ?$ & $5,10,11,12$ \\
\hline G241-6 & $\mathrm{He}$ & $\mathrm{N}$ & $\mathrm{S}, \mathrm{O}, \mathrm{Mn}, \mathrm{P}$ & $\mathrm{Cr}, \mathrm{Si}, \mathrm{Fe}, \mathrm{Mg}, \mathrm{Ni}$ & $\mathrm{Ca}, \mathrm{Ti}$ & primitive & $2,6,13$ \\
\hline HS $2253+8023$ & $\mathrm{He}$ & $\mathrm{N}$ & $\mathrm{O}, \mathrm{Mn}$ & $\mathrm{Cr}, \mathrm{Si}, \mathrm{Fe}, \mathrm{Mg}, \mathrm{Ni}:$ & $\mathrm{Ca}, \mathrm{Ti}$ & primitive & 6 \\
\hline
\end{tabular}

Note. This is a compiled sample of externally polluted white dwarfs with detections of at least 9 elements heavier than helium. Columns are defined as follows. "Dom" lists the dominant element in the atmosphere. "Dust" indicates whether a star has an infrared excess ("Y") or not ("N"). Following the classification scheme in Lodders (2003), "Volatile" lists the detected volatile elements, defined as having a $50 \%$ condensation temperature lower than $1290 \mathrm{~K}$ in a solar-system composition gas (Lodders 2003); "Intermediate" lists the elements with a condensation temperature between 1290-1360 K -the same range as that of the common elements, Si, Fe and Mg; "Refractory" elements have a $50 \%$ condensation temperature higher than $1360 \mathrm{~K}$. The elements are ordered with increasing condensation temperature. "Process" shows our proposed dominant mechanism that determines the final composition of the accreted extrasolar planetesimal (see section 5).

References. (1) Klein et al. (2010); (2) Jura et al. (2012); (3) Dufour et al. (2010); (4) Dufour et al. (2012); (5) Gänsicke et al. (2012); (6) Klein et al. (2011); (7) this paper; (8) Zuckerman et al. (2011); (9) Zuckerman et al. (2007); (10) Vennes et al. (2010); (11) Vennes et al. (2011); (12) Melis et al. (2011); (13) Zuckerman et al. (2010). 
$P G 0843+51 \%$ : This star has the highest mass fraction of iron among all polluted white dwarfs. Gänsicke et al. (2012) found that all core elements, including $\mathrm{Fe}, \mathrm{Ni}, \mathrm{S}$ and $\mathrm{Cr}$ are enhanced relative to the values for bulk Earth while lithophile refractory $\mathrm{Al}$ is depleted. This star might be accreting from the core of a differentiated object. Nevertheless, considering the uncertainty for each element is at least $0.2 \mathrm{dex}$, the conclusion is still preliminary.

$P G$ 1225-079: As discussed in section 4.2, this star has a near chondritic carbon abundance but also enhanced mass fractions of refractory elements relative to CI chondrite; it cannot be formed solely under nebular processing.

NLTT 43806: Compared to chondritic values, the accreted planetesimal is depleted in Fe and enhanced in Al. Zuckerman et al. (2011) found that the best fit model corresponds to " $30 \%$ crust $70 \%$ upper mantle". With detections of 9 elements, evidence is strong that NLTT 43806 has accreted the outer layer of a differentiated parent body.

GD 362: As discussed in section 4.1, mesosiderite is the best solar system analog to the accreted parent body and post-nebular processing is required.

WD 1929+012: Gänsicke et al. (2012) showed that this star has a high iron content. However, the situation is perplexing in that different analyses yield different stellar parameters and atmospheric abundances. For example, both Melis et al. (2011) and Gänsicke et al. (2012) derived that $[\mathrm{Si}] /[\mathrm{Fe}]$ is -0.25 but Vennes et al. (2010) found that $[\mathrm{Si}] /[\mathrm{Fe}]$ is 0.19 . No final conclusion can be drawn before resolving such discrepancies.

G241-6: This star is a near twin of GD 40 with a similar abundance pattern but without an infrared excess. One possible scenario is that G241-6 has accreted a planetesimal with a similar composition to GD 40 and now it is at the beginning of a decaying phase; all heavier elements appear to be depleted relative to GD 40 due to their short settling times (Klein et al. 2011; Jura et al. 2012). As discussed in Jura et al. (2012) and Appendix B, the overall abundances resemble those of chondrites and no post-nebular processing is required.

HS 2253+8023: Klein et al. (2011) showed that the composition of its parent body agrees with bulk Earth, except for the enhanced calcium abundance. Nebular processing can produce the observed abundance pattern.

As summarized in Table 6, at least 4 out of the 9 white dwarfs have accreted planetesimals that can be formed under nebular processing while post-nebular processing is required for another 3 of them. It should be noted that some objects that we identify as primitive might still have undergone some post-nebular processing. For example, GD 40 has accreted from a planetesimal that has a similar composition as bulk Earth, whose overall abundance pattern is chondritic. However, it is still possible that the parent body was differentiated; 
when the entire object is accreted, the composition appears to be "chondritic". We can only put an upper limit on the number of objects formed under nebular condensation.

From this sample of 9 stars, we see that post-nebular processing appears to play an important role in determining the final abundance of extrasolar planetesimals; beyondprimitive planetesimals might be as common as primitive planetesimals. In contrast, chondrites comprise more than $90 \%$ of all meteorites found on Earth by number (Meteorite Bulletin Databas $\left.9^{9}\right)$. Possibly, extrasolar planetesimals around white dwarfs have violent evolutionary histories with more collisions. This difference is not surprising since dynamical rearrangement of planetary systems at white dwarfs is expected to increase the frequency of collisions and produce more beyond-primitive extrasolar planetesimals.

So far, 19 elements heavier than helium, including C, S, O, Na, Cu, Mn, P, Cr, Si, Mg, $\mathrm{Fe}, \mathrm{Co}, \mathrm{Ni}, \mathrm{V}, \mathrm{Sr}, \mathrm{Ca}, \mathrm{Ti}, \mathrm{Al}$ and $\mathrm{Sc}$, have been detected in the atmospheres of polluted white dwarfs, as shown in Table 6. In terms of mass fraction in the accreted planetesimal, the lowest limit is $\sim 5 \mathrm{ppm}$, for Sc in WD J0738+1835 (Dufour et al. 2012). Studying externally-polluted white dwarfs proves to be a very sensitive probe of the bulk compositions of extrasolar planetesimals.

\section{CONCLUSIONS}

We present HST/COS ultraviolet observations for GD 362 and PG 1225-079, two heavily polluted helium white dwarfs. In GD 362, the mass fractions of carbon and sulfur are depleted by at least a factor of 7 and 3 respectively, compared to CI chondrites. In PG 1225-079, a similar volatile depletion pattern is found: $\mathrm{C}$ by a factor of $2, \mathrm{~S}$ by at least a factor of 40 and $\mathrm{Zn}$ by at least a factor of 8 . We provide good evidence for the presence of beyond-primitive extrasolar planetesimals:

1. Mesosiderites provide a good match to the composition of the parent body accreted onto GD 362. However, there are several unresolved issues for this hypothesis, especially the apparent difference between the mid-infrared spectrum of mesosiderites and the dust disk around GD 362. Additional material is required.

2. No single meteorite can reproduce the abundance pattern in PG 1225-079. A blend of $30 \%$ North Haig ureilite and 70\% Dyarrl Island mesosiderite can provide a good fit to the overall composition.

\footnotetext{
${ }^{9}$ http://www.lpi.usra.edu/meteor/
} 
3. Spectroscopic observations of externally-polluted white dwarfs enable sensitive measurement of the bulk compositions of extrasolar planetesimals, including 19 heavy elements down to a mass fraction of $5 \mathrm{ppm}$. Based on a sample of 9 well-studied white dwarfs, we find that post-nebular processing is as important as nebular condensation in determining the compositions of extrasolar planetesimals.

Support for program \# 12290 was provided by NASA through a grant from the Space Telescope Science Institute, which is operated by the Association of Universities for Research in Astronomy, Inc., under NASA contract NAS 5-26555. This work also has been partly supported by NSF grants to UCLA to study polluted white dwarfs.

\section{APPENDIX}

\section{A. The Herschel/PACS Observation of GD 362}

While hydrogen is detected in some helium-dominated white dwarfs (Voss et al. 2007), GD 362 has an anomalously large amount. The helium-to-hydrogen number ratio is 14 in its convective zone, corresponding to $5 \times 10^{24} \mathrm{~g}$ of hydrogen; this is lower than $7 \times 10^{24} \mathrm{~g}$ reported in Jura et al. (2009) because the mass of the convective zone for GD 362 is 0.13 dex lower in the updated calculation (Table 3). The origin of the hydrogen is a mystery. Unlike heavy elements which have short settling times compared to the white dwarf cooling age, hydrogen never sinks and can be accumulated over the entire cooling history of the star (Bergeron et al. 2011; Jura \& Xu 2012). If GD 362 has always been a helium-dominated white dwarf and all this hydrogen is from accretion of tidally disrupted objects, it can either be one Callisto-size object or $\sim 100$ Ceres-like asteroids (Jura et al. 2009). In the latter case, likely there would be many more asteroids orbiting the star and mutual collisions among them would generate a cloud of cold dust.

We were awarded 1.1 hours of Herschel/PACS (Poglitsch et al. 2010) observation time to look for cold dust around GD 362. The "mini-scan map" mode was used to observe in "blue" (85-125 $\mu \mathrm{m})$ and "red" (125-210 $\mu \mathrm{m})$ bands simultaneously with a medium scan speed of $20^{\prime \prime} \mathrm{s}^{-1}$ and a scan leg length of $4^{\prime}$. The scan map size is $345^{\prime \prime} \times 374^{\prime \prime}$ and the repetition number is 25. Two different scan angles, 45 degrees and 135 degrees were used and the total integration time was $1200 \mathrm{sec}$.

Data reduction was performed using HIPE (Herschel Interactive Processing Environment) on a combined mosaic of level 2 products from pipeline SPG 7.1.0. The pixel scale is $1^{\prime \prime}$ pixel $^{-1}$ and $2^{\prime \prime}$ pixel $^{-1}$ for the blue and red band, respectively. Correcting for its proper 
motion, we expect GD 362 at $\alpha=17: 31: 34.355, \delta=+37: 05: 18.331$ on the date of the observation. Because there is no detection, aperture photometry was performed at 25 locations within 5 pixels of the nominal position of GD 362. The aperture radius was $20^{\prime \prime}$ with a sky annulus between $61^{\prime \prime}$ and $70^{\prime \prime}$. The background intensity was estimated using the median sky estimation algorithm (Herschel Data Analysis Guide10). Aperture correction factors are 0.949 for blue and 0.897 for red (PACS Observer's Manual11). Based on the dispersion of the 25 measurements, $3 \sigma$ upper limits are $5.1 \mathrm{mJy}$ for blue and $5.6 \mathrm{mJy}$ for red.

What does this imply about dust mass? GD 362 has shrunk in mass from $3 \mathrm{M}_{\odot}$ on the main-sequence to its current mass of $0.72 \mathrm{M}_{\odot}$ (Kilic et al. 2008). Consequently, asteroids initially at 3-5 AU are now orbiting at 13-21 AU. Currently, GD 362 has a stellar temperature of $10,540 \mathrm{~K}$ and cooling age $\sim 0.9 \mathrm{Gyr}$ (Farihi et al. 2009). Extrapolating from white dwarf cooling models 12 (Bergeron et al. 2011), for GD 362, its stellar temperature is lower than $20,000 \mathrm{~K}$ for $90 \%$ of its cooling time. We approximate the stellar luminosity as a timeaveraged luminosity of $0.01 \mathrm{~L}_{\odot}$. Poynting-Robertson drag was able to remove particles smaller than $20 \mu \mathrm{m}$ at a distance of $15 \mathrm{AU}$ for a grain density of $3 \mathrm{~g} \mathrm{~cm}^{-3}$. We therefore assume a dust particle radius of $20 \mu \mathrm{m}$ in the putative asteroid belt orbiting GD 362 .

If the grains function as blackbodies with negligible albedo, then their temperature can be calculated as

$$
T_{d}=T_{*} \sqrt{\frac{R_{*}}{2 D_{o r b}}}
$$

$\mathrm{T}_{*}, \mathrm{R}_{*}$ are the stellar temperature and radius; $\mathrm{D}_{\text {orb }}$ is the orbital distance. The dust temperature is 14-11 $\mathrm{K}$ between 13-21 AU.

The mass of the dust disk is

$$
M_{d}=\frac{F_{\nu} D_{*}^{2}}{\chi B_{\nu}(T)}
$$

where $\mathrm{D}_{*}$ is the distance to GD 362, $51 \mathrm{pc}$ (Kilic et al. 2008) and $\chi$ is the dust opacity. For a particle radius of $20 \mu \mathrm{m}, \chi=100 \mathrm{~cm}^{2} \mathrm{~g}^{-1}$ in the geometric optics limit. As shown in Figure 11, the upper limit of dust mass is between $10^{25} \mathrm{~g}$ and $10^{26} \mathrm{~g}$ at $13-21 \mathrm{AU}$; this mass is at

\footnotetext{
${ }^{10}$ http://herschel.esac.esa.int/hcss-doc-8.0/print/howtos/howtos.pdf

${ }^{11}$ http://herschel.esac.esa.int/Docs/PACS/pdf/pacs_om.pdf

${ }^{12}$ http://www.astro.umontreal.ca/ bergeron/CoolingModels/
} 
least twice the hydrogen mass in the atmosphere of GD 362 and one order of magnitude larger than the mass of solar system's asteroid belt (Krasinsky et al. 2002). The upper limit is not stringent enough to rule out the hypothesis that hydrogen in GD 362 is from accretion of multiple asteroids. So, the large hydrogen abundance in GD 362 remains an unsolved puzzle.

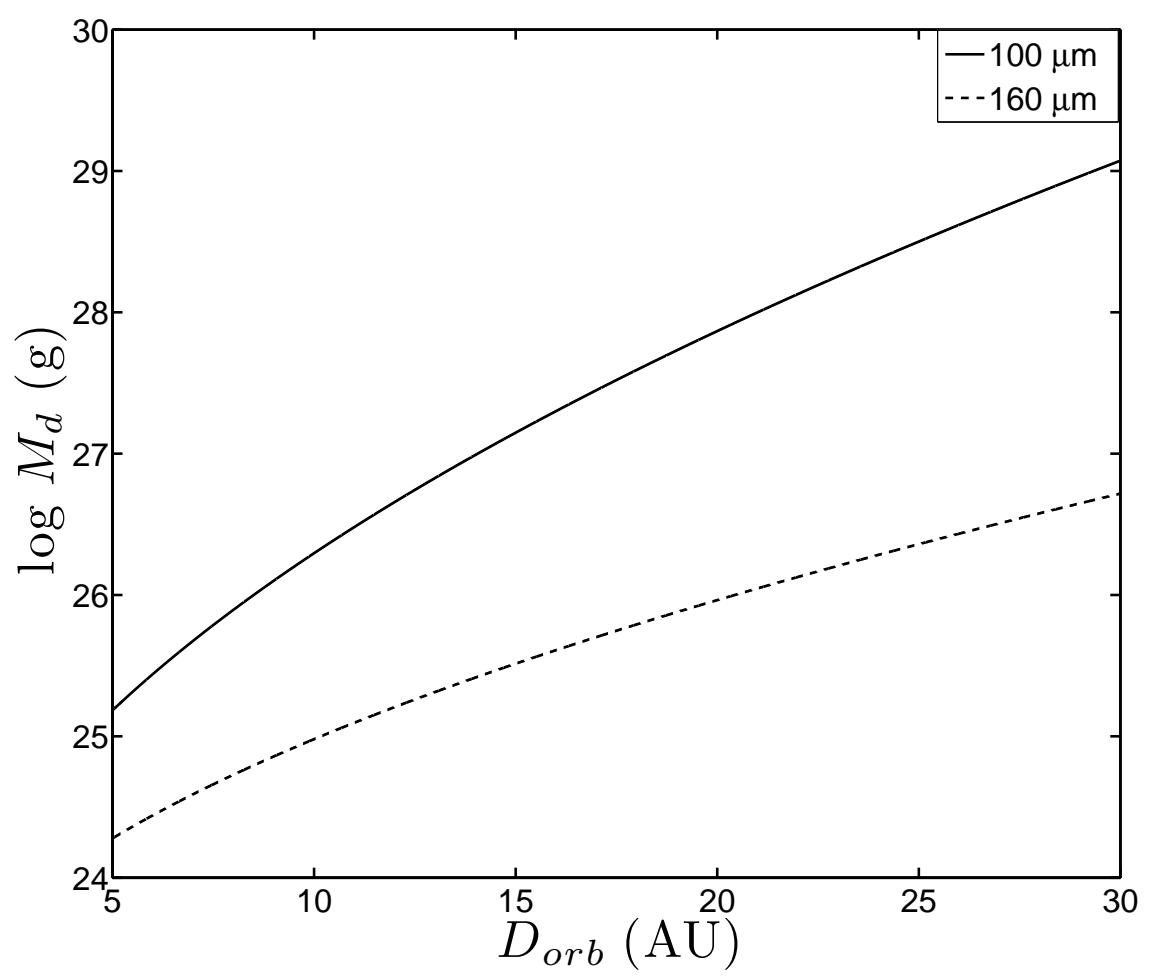

Fig. 11. - Upper limit for the mass of cold dust around GD 362 derived from PACS blue and red data, as a function of orbital radius. Given the hydrogen mass of $5 \times 10^{24} \mathrm{~g}$, the upper limit of dust mass $10^{25}-10^{26} \mathrm{~g}$ at $13-21 \mathrm{AU}$ is not stringent enough to rule out the accretion of multiple asteroids.

\section{B. Looking for Solar System Analogs to Extrasolar Planetesimals}

The $\chi_{\text {red }}^{2}$ analysis has proven to be an effective way to look for solar system analogs to the compositions of extrasolar planetesimals. Two other helium-dominated white dwarfs have reported volatile and refractory abundances from high-resolution optical and ultraviolet 
observations that are suitable for this kind of analysis 13 - GD 40 and G241-6. Updated settling times and accretion rates are listed in Table 7 while the mass of the convective zone stays the same. Since all the major elements are determined, we compare the mass fraction of an element relative to the sum of $\mathrm{O}, \mathrm{Mg}$, Si and Fe.

Table 7: Updated Settling Times and Accretion Rates for GD 40 and G241-6

\begin{tabular}{|c|c|c|c|}
\hline Z & $\begin{array}{l}\mathrm{t}_{\text {set }}{ }^{a} \\
\left(10^{6} \mathrm{yr}\right)\end{array}$ & $\begin{array}{l}\dot{M}(\mathrm{Z})_{G D 40} \\
\left(\mathrm{~g} \mathrm{~s}^{-1}\right)\end{array}$ & $\begin{array}{c}M(\mathrm{Z})_{G 241-6} \\
\quad\left(\mathrm{~g} \mathrm{~s}^{-1}\right)\end{array}$ \\
\hline $\mathrm{C}$ & 1.1 & $2.2 \times 10^{6}$ & $<4.4 \times 10^{5}$ \\
\hline $\mathrm{N}$ & 1.1 & $<2.6 \times 10^{5}$ & $<2.1 \times 10^{5}$ \\
\hline $\mathrm{O}$ & 1.1 & $4.5 \times 10^{8}$ & $4.3 \times 10^{8}$ \\
\hline $\mathrm{Mg}$ & 1.2 & $1.7 \times 10^{8}$ & $1.5 \times 10^{8}$ \\
\hline $\mathrm{Al}$ & 1.2 & $1.4 \times 10^{7}$ & $<6.1 \times 10^{6}$ \\
\hline $\mathrm{Si}$ & 1.0 & $1.3 \times 10^{8}$ & $8.7 \times 10^{7}$ \\
\hline $\mathrm{P}$ & 0.79 & $1.1 \times 10^{6}$ & $4.7 \times 10^{5}$ \\
\hline S & 0.64 & $1.0 \times 10^{7}:$ & $5.6 \times 10^{7}$ \\
\hline $\mathrm{Cl}$ & 0.51 & $<8.0 \times 10^{5}$ & $<5.8 \times 10^{5}$ \\
\hline $\mathrm{Ca}$ & 0.51 & $1.3 \times 10^{8}$ & $5.1 \times 10^{7}$ \\
\hline $\mathrm{Ti}$ & 0.49 & $3.2 \times 10^{6}$ & $1.4 \times 10^{6}$ \\
\hline $\mathrm{Cr}$ & 0.53 & $6.4 \times 10^{6}$ & $4.5 \times 10^{6}$ \\
\hline Mn & 0.53 & $3.1 \times 10^{6}$ & $2.4 \times 10^{6}$ \\
\hline $\mathrm{Fe}$ & 0.56 & $4.4 \times 10^{8}$ & $2.0 \times 10^{8}$ \\
\hline $\mathrm{Ni}$ & 0.61 & $1.8 \times 10^{7}$ & $8.9 \times 10^{6}$ \\
\hline $\mathrm{Cu}$ & 0.58 & $<1.8 \times 10^{5}$ & $<1.8 \times 10^{5}$ \\
\hline $\mathrm{Ga}$ & 0.50 & $<2.9 \times 10^{4}$ & $<2.9 \times 10^{4}$ \\
\hline $\mathrm{Ge}$ & 0.43 & $<1.4 \times 10^{5}$ & $<1.4 \times 10^{5}$ \\
\hline Total & & $1.4 \times 10^{9}$ & $9.9 \times 10^{8}$ \\
\hline
\end{tabular}

${ }^{a}$ This column is for both GD 40 and G241-6 because their atmospheric conditions are similar.

The total accretion rate for GD 40 is a factor of 2 lower than the value derived in Klein et al. (2010), but the relative abundances change much less. The result of a $\chi_{\text {red }}^{2}$ analysis is presented in Figure 12. When including all 13 detected elements, both carbonaceous chondrites and bulk Earth can match the composition to $95 \%$ confidence level for

\footnotetext{
${ }^{13}$ GD 61 also has high-resolution optical and ultraviolet observations (Desharnais et al. 2008; Farihi et al. 2011). However, with a total of 5 detected elements, it is hard to make a comparison using the $\chi_{\text {red }}^{2}$ analysis.
} 
both steady-state and build-up approximations. The accreted planetesimal appears to be primitive and can be formed under nebular condensation, similar to what was concluded by Jura et al. (2012).

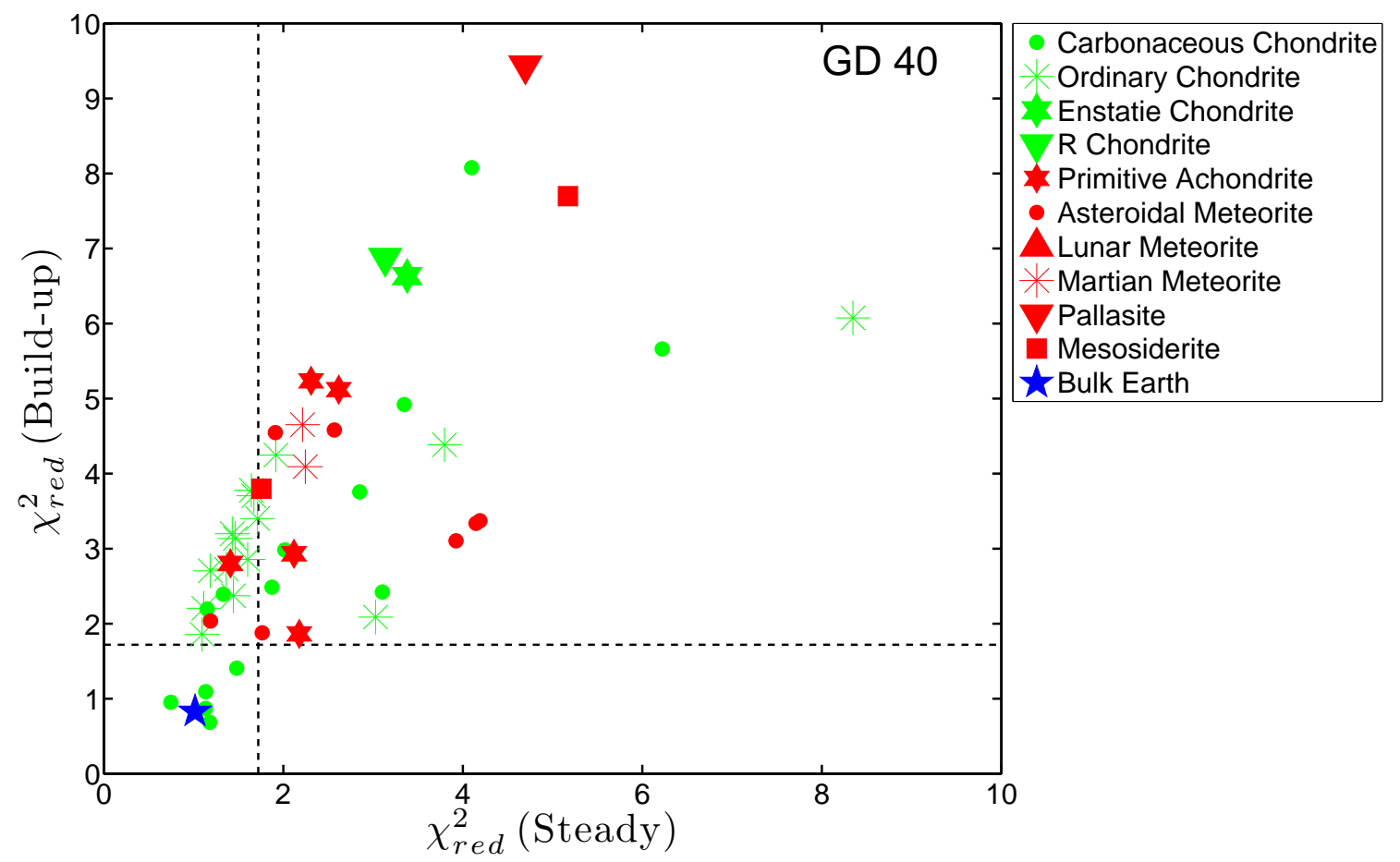

Fig. 12.- Similar to Figure 7 except for GD 40 comparing the mass fraction of 13 elements, including C, O, Mg, Al, Si, P, S, Ca, Ti, Cr, Mn, Fe and Ni, relative to the sum of Mg, Si, Fe and O. Both carbonaceous chondrites and bulk Earth are a good match to the parent body accreted onto GD 40.

The newly-derived total accretion rate for G241-6 is about a factor of 2 lower than previously reported (Zuckerman et al. 2010). The non-detection of an infrared excess and the slight depletion of heavier elements suggest that it may be at the beginning of a decay phase (Xu \& Jura 2012; Klein et al. 2011). We assess both steady-state and decay phase for the $\chi_{\text {red }}^{2}$ analysis; in the latter case, we assume that accretion stopped $0.6 \times 10^{6} \mathrm{yr}$ ago, approximately one settling time for Fe because its mass fraction is depleted by a factor of 2 relative to CI chondrites. The composition of the parent body is calculated following Zuckerman et al. (2011) and Equation (5) in Koester (2009). A fuller exploration of different time-varying models will be presented in the future in the spirit of Jura \& Xu (2012). As shown in Figure 13, both carbonaceous chondrites and ordinary chondrites provide good matches to all 11 elements, including O, Mg, Si, P, S, Ca, Ti, Cr, Mn, Fe and Ni. However, the 
carbon upper limit in G241-6, which is not included in the $\chi_{\text {red }}^{2}$ analysis, is at least one order of magnitude lower than most carbonaceous chondrites (Jura et al. 2012). Thus, ordinary chondrites are a more promising solar system analog to the parent body accreted onto G241-6 and nebular condensation is sufficient to produce the observed abundance pattern.

The $\chi_{\text {red }}^{2}$ analysis for GD 40 and G241-6 confirms the previous results (Jura et al. 2012); the accreted extrasolar planetesimals can be formed under nebular condensation and their compositions resemble primitive chondrites in the solar system.

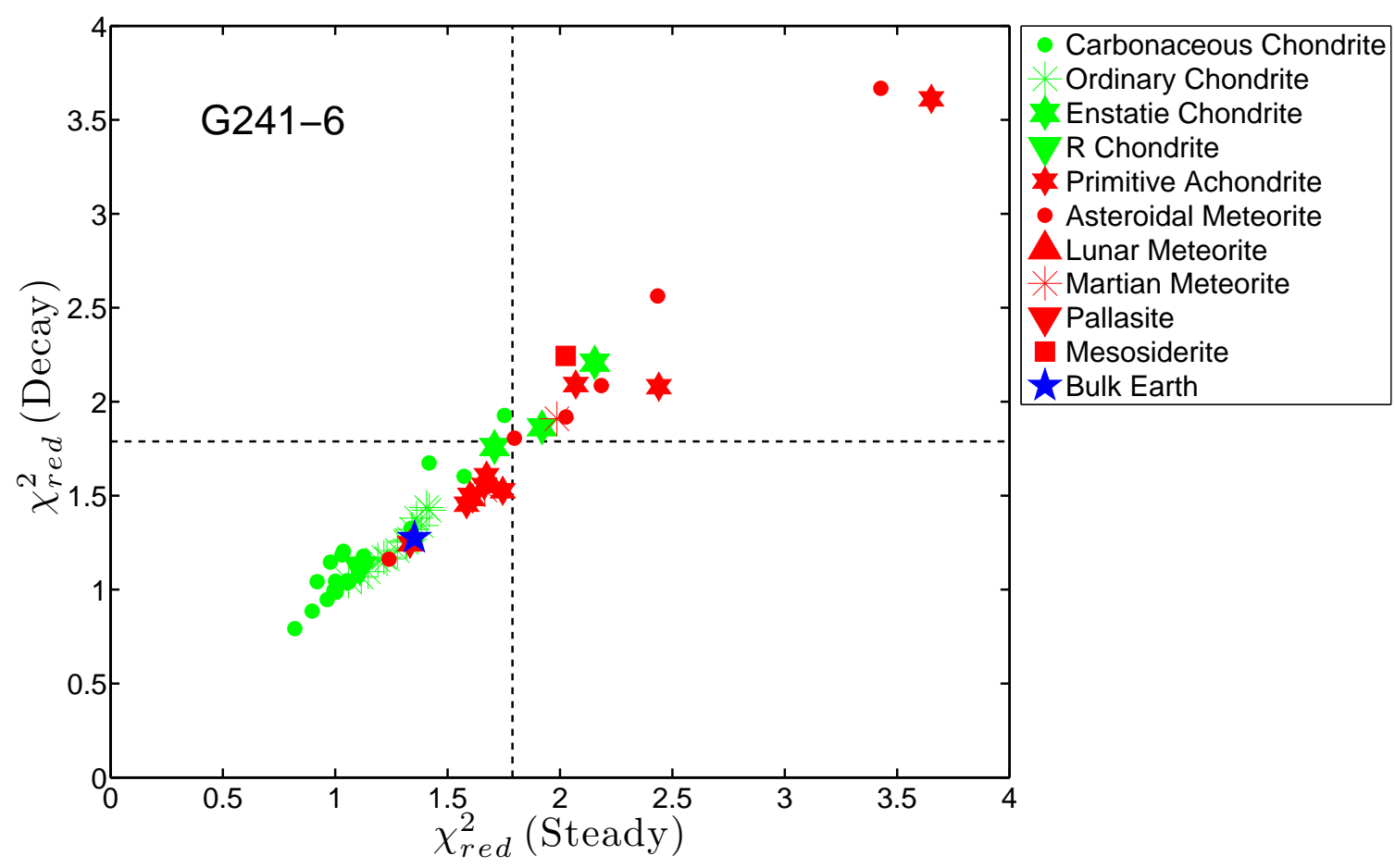

Fig. 13.- Similar to Figure 12 except for G241-6 comparing 11 elements - O, Mg, Si, P, S, $\mathrm{Ca}, \mathrm{Ti}, \mathrm{Cr}, \mathrm{Mn}, \mathrm{Fe}$ and $\mathrm{Ni}$ for steady-state versus decay phase when the accretion stopped $0.6 \times 10^{6}$ yr ago. Both carbonaceous chondrites and ordinary chondrites produce a good fit to the parent body accreted onto G241-6. Ordinary chondrites are a relatively better match because of the low carbon abundance, which is not considered in this $\chi_{\text {red }}^{2}$ plot because only an upper limit was reported for G241-6 (Jura et al. 2012).

\section{REFERENCES}

Allègre, C., Manhès, G., \& Lewin, E. 2001, Earth and Planetary Science Letters, 185, 49 
Barber, S. D., Patterson, A. J., Kilic, M., Leggett, S. K., Dufour, P., Bloom, J. S., \& Starr, D. L. 2012, ApJ, 760, 26

Becklin, E. E., Farihi, J., Jura, M., Song, I., Weinberger, A. J., \& Zuckerman, B. 2005, ApJ, 632, L119

Benz, W., Slattery, W. L., \& Cameron, A. G. W. 1988, Icarus, 74, 516

Bergeron, P., Wesemael, F., Dufour, P., Beauchamp, A., Hunter, C., Saffer, R. A., Gianninas, A., Ruiz, M. T., Limoges, M.-M., Dufour, P., Fontaine, G., \& Liebert, J. 2011, ApJ, 737,28

Bonsor, A., Mustill, A. J., \& Wyatt, M. C. 2011, MNRAS, 594

Chyba, C. F. 1990, Nature, 343, 129

Debes, J. H. \& Sigurdsson, S. 2002, ApJ, 572, 556

Debes, J. H., Walsh, K. J., \& Stark, C. 2012, ApJ, 747, 148

Desharnais, S., Wesemael, F., Chayer, P., Kruk, J. W., \& Saffer, R. A. 2008, ApJ, 672, 540

Dufour, P., Kilic, M., Fontaine, G., Bergeron, P., Lachapelle, F., Kleinman, S. J., \& Leggett, S. K. 2010, ApJ, 719, 803

Dufour, P., Kilic, M., Fontaine, G., Bergeron, P., Melis, C., \& Bochanski, J. 2012, ApJ, 749, 6

Dupuis, J., Fontaine, G., Pelletier, C., \& Wesemael, F. 1993, ApJS, 84, 73

Farihi, J., Becklin, E. E., \& Zuckerman, B. 2005, ApJS, 161, 394

Farihi, J., Brinkworth, C. S., Gänsicke, B. T., Marsh, T. R., Girven, J., Hoard, D. W., Klein, B., \& Koester, D. 2011, ApJ, 728, L8

Farihi, J., Gänsicke, B. T., Wyatt, M. C., Girven, J., Pringle, J. E., \& King, A. R. 2012, MNRAS, 424, 464

Farihi, J., Jura, M., Lee, J., \& Zuckerman, B. 2010, ApJ, 714, 1386

Farihi, J., Jura, M., \& Zuckerman, B. 2009, ApJ, 694, 805

Gänsicke, B. T., Koester, D., Farihi, J., Girven, J., Parsons, S. G., \& Breedt, E. 2012, MNRAS, 424, 333 
Girven, J., Brinkworth, C. S., Farihi, J., Gänsicke, B. T., Hoard, D. W., Marsh, T. R., \& Koester, D. 2012, ApJ, 749, 154

Goodrich, C. A. 1992, Meteoritics, 27, 327

Jura, M. 2003, ApJ, 584, L91

-. 2008, AJ, 135, 1785

-. 2013, ArXiv e-prints

Jura, M., Farihi, J., Zuckerman, B., \& Becklin, E. E. 2007, AJ, 133, 1927

Jura, M., Muno, M. P., Farihi, J., \& Zuckerman, B. 2009, ApJ, 699, 1473

Jura, M. \& Xu, S. 2010, AJ, 140, 1129

-. 2012, AJ, 143, 6

-. 2013, AJ, in press

Jura, M., Xu, S., Klein, B., Koester, D., \& Zuckerman, B. 2012, ApJ, 750, 69

Kelleher, D. \& Podobedova, L. 2008, JPCRD, 37, 267

Kilic, M., Thorstensen, J. R., \& Koester, D. 2008, ApJ, 689, L45

Kilic, M., von Hippel, T., Leggett, S. K., \& Winget, D. E. 2005, ApJ, 632, L115

-. 2006, ApJ, 646, 474

Klein, B., Jura, M., Koester, D., \& Zuckerman, B. 2011, ApJ, 741, 64

Klein, B., Jura, M., Koester, D., Zuckerman, B., \& Melis, C. 2010, ApJ, 709, 950

Koester, D. 2009, A\&A, 498, 517

—. 2010, Mem. Soc. Astron. Italiana, 81, 921

Koester, D., Gänsicke, B., Girven, J., \& Farihi, J. 2012, ArXiv e-prints 1206:6036

Krasinsky, G. A., Pitjeva, E. V., Vasilyev, M. V., \& Yagudina, E. I. 2002, Icarus, 158, 98

Kupka, F., Piskunov, N., Ryabchikova, T. A., Stempels, H. C., \& Weiss, W. W. 1999, A\&AS, 138, 119 
Larimer, J. W. 1988, Meteorites and the Early Solar System (University of Arizona Press), 19-52

Lodders, K. 2003, ApJ, 591, 1220

Marty, B. 2012, Earth and Planetary Science Letters, 313, 56

McSween, H. Y. 1985, Reviews of Geophysics, 23, 391

Melis, C., Farihi, J., Dufour, P., Zuckerman, B., Burgasser, A. J., Bergeron, P., Bochanski, J., \& Simcoe, R. 2011, ApJ, 732, 90

Morlok, A., Koike, C., Tomeoka, K., Mason, A., Lisse, C., Anand, M., \& Grady, M. 2012, Icarus, 219, 48

Morton, D. C. 1975, ApJ, 197, 85

Nittler, L. R., McCoy, T. J., Clark, P. E., Murphy, M. E., Trombka, J. I., \& Jarosewich, E. 2004, Antarctic Meteorite Research, 17, 231

O’Neill, H. S. C. \& Palme, H. 2008, Royal Society of London Philosophical Transactions Series A, 366, 4205

Paquette, C., Pelletier, C., Fontaine, G., \& Michaud, G. 1986, ApJS, 61, 197

Pelletier, C., Fontaine, G., Wesemael, F., Michaud, G., \& Wegner, G. 1986, ApJ, 307, 242

Poglitsch, A., Waelkens, C., Geis, N., \& et al. 2010, A\&A, 518, L2

Rafikov, R. R. 2011a, ApJ, 732, L3+

-. 2011b, MNRAS, 416, L55

Scott, E. R. D., Haack, H., \& Love, S. G. 2001, Meteoritics and Planetary Science, 36, 869

Vennes, S., Kawka, A., \& Németh, P. 2010, MNRAS, 404, L40

-. 2011, MNRAS, 413, 2545

von Hippel, T., Kuchner, M. J., Kilic, M., Mullally, F., \& Reach, W. T. 2007, ApJ, 662, 544

Voss, B., Koester, D., Napiwotzki, R., Christlieb, N., \& Reimers, D. 2007, A\&A, 470, 1079

Warren, P. H., Ulff-Møller, F., Huber, H., \& Kallemeyn, G. W. 2006, Geochim. Cosmochim. Acta, 70, 2104 
Wolff, B., Koester, D., \& Liebert, J. 2002, A\&A, 385, 995

Xu, S. \& Jura, M. 2012, ApJ, 745, 88

Zuckerman, B., Koester, D., Dufour, P., Melis, C., Klein, B., \& Jura, M. 2011, ApJ, 739, 101

Zuckerman, B., Koester, D., Melis, C., Hansen, B. M., \& Jura, M. 2007, ApJ, 671, 872

Zuckerman, B., Melis, C., Klein, B., Koester, D., \& Jura, M. 2010, ApJ, 722, 725 